\title{
Hausdorff dimension of real numbers with bounded digit averages
}

\author{
by \\ Eda Cesaratto (Buenos Aires and Caen) and \\ Brigitte Vallée (Caen)
}

\section{INTRODUCTION}

A numeration process associates to each real number $x$ of the unit interval $I$ a sequence of digits $\left(m_{1}(x), m_{2}(x), \ldots\right)$ where each $m_{i}$ belongs to some alphabet $\mathcal{M} \subset \mathbb{N}^{\star}$, finite or denumerable. The numeration in base $b$ and the continued fraction expansion are the most studied instances of such processes which are defined in terms of dynamical systems. A dynamical system (on the interval) is a pair $(I, T)$ formed by an interval $I$ and a map $T: I \rightarrow I$. There exist a topological partition $I_{m}$ of $I$ indexed by the alphabet $\mathcal{M}$, and a coding map $\varrho: I \rightarrow \mathcal{M}$ which is constant and equal to $m$ on the interval $I_{m}$, so that the digit $m_{i}(x):=\varrho\left(T^{i-1} x\right)$ is the index of the interval to which the iterate $T^{i-1} x$ belongs. Then one associates to each real $x$ of $I$ the word

$$
\left(m_{1}(x), m_{2}(x), \ldots\right) \quad \text { where } \quad m_{i}(x)=\varrho\left(T^{i-1} x\right),
$$

which provides a useful coding of the trajectory of $x$ under the map $T$,

$$
\mathcal{T}(x):=\left(x, T x, T^{2} x, \ldots\right) .
$$

Moreover, one assumes that each branch of $T$, i.e., the restriction of $T$ to each $I_{m}$, is strictly monotone, with $T\left(I_{m}\right)=I$. Its inverse is denoted by $h_{m}$; the set of inverse branches of $T$ is denoted by $\mathcal{H}$. In this paper, we study dynamical systems where the set $\mathcal{H}$ has nice properties; they all belong to the so-called Good Class defined in Section 2.1. This framework provides numeration processes where the $n$th digit $m_{n}$ may depend on the whole previous history.

2000 Mathematics Subject Classification: 11A55, 11K50, 11K55, 11A63, 37A45, 37E05, 37C30, 60F 10 .

Key words and phrases: dynamical systems, transfer operator, Hausdorff dimension, quasi-powers theorem, large deviations, shifting of the mean, Mellin analysis. 
Elementary constraints on numeration processes. In this setting, it is now classical to study numbers $x$ for which the sequence (1.1) satisfies some particular constraints. The instance of Cantor sets where the constraint is the same for each digit $m_{i}$ and only allows a subset $\mathcal{A}$ of possible values is well known. In this case, the set $E_{\mathcal{A}}$ of such constrained numbers has zero measure, and it is thus of great interest to study its Hausdorff dimension. The first study on the subject concerns numeration in base $b$ and is due to Eggleston [13]. The problem is now completely solved when the alphabet is finite.

The case of an infinite alphabet (even if the process is memoryless) is a little more difficult, and the properties of the set $\mathcal{A}$ of constraints have to be made precise $[35,36]$. In a quite general setting (dynamical systems of the Good Class, "open" constraints), the question is solved. The case when the alphabet is infinite is quite important since it contains a particular case of great interest: the reals whose continued fraction expansion only contains digits $m_{i}$ less than $M$. These reals are badly approximable by rationals, and appear in many contexts of number theory (see [31, 40]).

The main tool is a constrained version of the transfer operator relative to the dynamical system $(I, T)$. The transfer operator $\mathbf{H}_{s}$, defined as

$$
\mathbf{H}_{s}[f]:=\sum_{m \in \mathcal{M}}\left|h_{m}^{\prime}\right|^{s} \cdot f \circ h_{m},
$$

involves the set $\mathcal{H}$ of inverse branches of $T$ and extends the density transformer $\mathbf{H}$ which describes the evolution of the density during the iterations of the dynamical system. When $s$ equals 1 , the operator $\mathbf{H}_{s}$ coincides with $\mathbf{H}$. The constrained transfer operator (relative to the set $\mathcal{A}$ of constraints) $\mathbf{H}_{\mathcal{A}, s}$ is then defined by

$$
\mathbf{H}_{\mathcal{A}, s}[f]:=\sum_{m \in \mathcal{A}}\left|h_{m}^{\prime}\right|^{s} \cdot f \circ h_{m} .
$$

For a dynamical system of the Good Class, and for real values of $s$, the operator $\mathbf{H}_{\mathcal{A}, s}$ has a unique dominant eigenvalue denoted by $\lambda_{\mathcal{A}}(s)$. When the set $\mathcal{A}$ is "open", there exists a (unique) real $s=\tau_{\mathcal{A}}$ for which $\lambda_{\mathcal{A}}(s)=1$ and the Hausdorff dimension of $E_{\mathcal{A}}$ equals $\tau_{\mathcal{A}}$.

The particular case of "constrained" continued fractions was extensively studied; the beginners were Jarník [28, 29], Besicovitch [5] and Good [17]. Then Cusick [11], Hirst [24] and Bumby [9] brought important contributions, and finally Hensley [19-22] completely solved the problem. In [41], this result was extended to the case of "periodic" constraints.

Another question of interest is the asymptotic behaviour of $\operatorname{dim} E_{\mathcal{A}}$ when the constraint becomes weaker (i.e., $\mathcal{A} \rightarrow \mathcal{M}$ ). Then the Hausdorff dimension tends to 1 , and the speed of convergence towards 1 is also an important question. In the case of continued fractions, Hensley [21] studies the 
case when $\mathcal{A}_{M}:=\{1, \ldots, M\}$ and exhibits the asymptotic behaviour of $\tau_{M}:=\operatorname{dim} E_{\mathcal{A}_{M}}$ when $M \rightarrow \infty$ :

$$
\left|\tau_{M}-1\right|=\frac{6}{\pi^{2}} \frac{1}{M}+O\left(\frac{\log M}{M^{2}}\right)
$$

Bounded prefix averages. We consider here constraints which are more general than the previous ones. They are defined by conditions which only bound all the weighted prefix averages. Define a $\operatorname{cost}$ (or weight) $c: \mathcal{M} \rightarrow \mathbb{R}^{+}$ on the digits. On each truncated trajectory $\mathcal{T}_{n}(x)$ encoded by the $n$-uple $\left(m_{1}(x), \ldots, m_{n}(x)\right)$ defined in (1.1), define the total cost $C_{n}(x)$ and the weighted prefix average $M_{n}(x)$ as

$$
C_{n}(x):=\sum_{i=1}^{n} c\left(m_{i}(x)\right), \quad M_{n}(x):=\frac{1}{n} C_{n}(x) .
$$

For any $M>0$, consider the set $F_{M}$ formed by the reals $x$ for which each weighted prefix average $M_{n}(x)$ is bounded by $M$, and the set $\widetilde{F}_{M}$ of reals $x$ for which $M_{n}(x) \leq M$ for $n$ sufficiently large. The sets $F_{M}, \widetilde{F}_{M}$ can also be described in terms of random walks. To each real number $x$, one associates the walk formed by points $\left(P_{i}(x)\right)_{i \geq 0}$. One begins with $P_{0}(x):=(0,0)$, and, at time $i$, one performs a step $P_{i}(x)-P_{i-1}(x):=\left(1, c\left(m_{i}(x)\right)\right)$. Then $F_{M}$ is the set of reals $x$ for which the walk $\left(P_{i}(x)\right)_{i \geq 0}$ is always under the line of slope $M$, while $\widetilde{F}_{M}$ gathers the reals $x$ for which the walk $\left(P_{i}(x)\right)_{i \geq 0}$ is ultimately under the line of slope $M$.

The strength of these constraints clearly depends on the relation between the cost $c$ and the occurrence probability of the digits. For a dynamical system of the Good Class, with an infinite alphabet $\mathcal{M}$, consider the (initial) probability distribution $p: k \mapsto p_{k}$ of digit $m_{1}$, together with the limit distribution $\bar{p}$ of the $n$th digit $m_{n}$ (as $\left.n \rightarrow \infty\right)$ which always exists in the Good Class setting:

$$
p_{k}:=\mathbb{P}\left[m_{1}(x)=k\right], \quad \bar{p}_{k}:=\lim _{n \rightarrow \infty} \mathbb{P}\left[m_{n}(x)=k\right] .
$$

We are mostly interested in the case when the sequence $m \mapsto p_{m}$ is decreasing, and the sequence $m \mapsto c(m)$ is strictly increasing to $+\infty$, with a minimal cost $\gamma(c)=c(1)>0$. The mixed sequences

$$
\pi_{n}:=\min \left\{p_{m} ; c(m) \leq n\right\} \quad \text { and } \quad \bar{\pi}_{n}:=\min \left\{\bar{p}_{m} ; c(m) \leq n\right\}
$$

summarize the balance between the increase of the cost $c$ and the decrease of the distributions $p, \bar{p}$, and the conditions

$$
\liminf \pi_{n}^{1 / n}=1 \quad \text { and } \quad \liminf \bar{\pi}_{n}^{1 / n}=1
$$

(which are equivalent for systems of the Good Class) informally express that the increase of $c$ is faster than the decrease of $p$. In this case, the triple $(I, T, c)$ is said to be of large growth $(\mathcal{G} \mathcal{L G}$-setting $)$. In the opposite case, it is 
said to be of moderate growth $(\mathcal{G M G}$-setting). The moderate growth setting

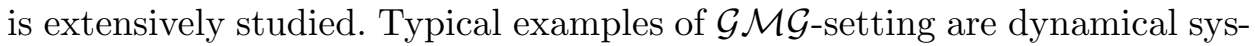
tems with a finite number of branches, where the cost function cannot be of large growth. A classical instance of the $\mathcal{G M G}$-setting is the $b$-ary expansion. The particular case when $b=2$ and $c(m)=m$ was studied by Besicovitch [6] and Eggleston [13] and their results are well described in [14]. The authors proved that the Hausdorff dimension of the set $F_{M}$ (for $M \leq 1 / 2$ ) equals

$$
-M \log _{2} M-(1-M) \log _{2}(1-M) .
$$

Here, we focus on the large growth setting. To the best of our knowledge, the $\mathcal{G} \mathcal{L} \mathcal{G}$-setting has been less studied, and we are mainly interested in a particular case of it, namely the continued fractions expansions where all the prefix digit averages are bounded.

Consider the (stationary) average $\mu(c)$ of the cost $c$ (possibly infinite)

$$
\mu(c):=\sum_{m=1}^{\infty} c(m) \bar{p}_{m} .
$$

When $M>\gamma(c)$, the set $F_{M}$ is not empty. When $M<\mu(c)$, the sets $F_{M}, \widetilde{F}_{M}$ have zero measure. In order to get more precise information on $F_{M}, \widetilde{F}_{M}$, it is thus of great interest to study their Hausdorff dimension. It is easy to prove that these two sets have the same Hausdorff dimension, denoted by $s_{M}$. We first wish to provide a (mathematical) characterization of $s_{M}$. In most cases, when $M$ tends to $\mu(c)$, the dimension $s_{M}$ tends to 1 and we also wish to obtain the exact asymptotic behaviour of $\left|s_{M}-1\right|$.

Continued fractions with bounded digit averages. In the case when $c(m)$ $=m$, the random variable $C_{n}=n M_{n}$ defined in (1.5) equals the sum of the first $n$ digits of the continued fraction expansion and is extensively studied. Its limit distribution is a quasi-stable law. Hensley [23] studied the measure $a_{n}(M)$ of reals $x$ for which $M_{n}(x) \leq M$, and exhibited the asymptotic behaviour of $a_{n}(M)$ as $n \rightarrow \infty$ uniformly in $M$ : there exists an explicit function $a(M)$ (which tends to 0 as $M \rightarrow+\infty$ ) for which

$$
\lim _{n \rightarrow \infty} \sup _{M}\left|a_{n}(M)-a(M)\right|=0 .
$$

However, to the best of our knowledge, the set $F_{M}$ of reals for which all the $M_{n}(x)$ are less than $M$ has not yet been studied, and it brings some precise and complementary information on the subject.

Main tools and main results. As in previous works on related subjects $[18,3,4]$, our main tool is the weighted transfer operator $\mathbf{H}_{s, w}$ related to the triple $(I, T, c)$,

$$
\mathbf{H}_{s, w}[f]:=\sum_{h \in \mathcal{H}} \exp [w c(h)] \cdot\left|h^{\prime}\right|^{s} \cdot f \circ h,
$$


where $s$ and $w$ are complex parameters. The precise values for which $\mathbf{H}_{s, w}$ is well defined are made precise in Section 4.2. (Since the set $\mathcal{H}$ of inverse branches is indexed by the digits, the cost $c$ is also defined on $\mathcal{H}$.) This weighted operator extends the transfer operator $\mathbf{H}_{s}$ already defined in (1.2), and when $w=0$, the operator $\mathbf{H}_{s, w}$ coincides with $\mathbf{H}_{s}$. On a convenient function space, and for real values of $s$ and $w$, this operator has a unique dominant eigenvalue $\lambda(s, w)$, and the pressure function is $\Lambda(s, w):=\log \lambda(s, w)$. The analysis involves the behaviour of $\Lambda(s, w)$ when $(s, w)$ is near the reference point $(1,0)$. For a triple of $\mathcal{G} \mathcal{L G}$-type, the map $(s, w) \mapsto \Lambda(s, w)$ is not analytic at $(1,0)$, whereas it is analytic at $(1,0)$ for a triple of $\mathcal{G M \mathcal { G }}$-type. Note that all the previous analysis $[18,3,4]$ dealt with $\mathcal{G M G}$-triples, and heavily used the analyticity at $(1,0)$.

We obtain two main results. The first theorem provides a (mathematical) characterization of the Hausdorff dimension $s_{M}$ of $F_{M}$ as a solution of an implicit system.

Theorem 1. Consider the set $F_{M}$ relative to a triple $(I, T, c)$ of $\mathcal{G} \mathcal{L G}$ type. Denote by $\gamma(c)$ the minimal value of the cost $c$, and by $\mu(c)$ the stationary average of $c$. Denote by $\mathbf{H}_{s, w}$ the weighted operator relative to the triple $(I, T, c)$ defined in (1.7) and by $\Lambda(s, w)$ the logarithm of its dominant eigenvalue when $\mathbf{H}_{s, w}$ acts on $\mathcal{C}^{1}(I)$. Then, for any $\gamma(c)<M<\mu(c)$, there exists a unique pair $\left.\left(s_{M}, w_{M}\right) \in[0,1] \times\right]-\infty, 0[$ for which

$$
(\mathcal{S}): \quad \Lambda(s, w)=M w, \quad \frac{\partial}{\partial w} \Lambda(s, w)=M,
$$

and $s_{M}$ is the Hausdorff dimension of $F_{M}$. Moreover, the two functions $M \mapsto s_{M}, M \mapsto w_{M}$ are analytic at any point $\left.M \in\right] \gamma(c), \mu(c)[$.

We then come back to our main motivation: the numeration process related to continued fraction expansion, and the set $F_{M}$ of reals for which all the digit averages are bounded by $M$. This triple is a particular case of what we call a boundary triple, i.e., one for which the series $\sum_{m \geq 1} c(m) p_{m}^{s}$ has convergence abscissa equal to 1 . Here, we consider a subclass of boundary triples, the so-called Dirichlet boundary triples, for which some Dirichlet series (which involve both the probability $p$ and cost $c$ ) have nice properties (see Definition 6 in Section 6). Important instances of Dirichlet boundary triples are memoryless sources of a Riemann type, denoted by $\mathcal{B R}(\alpha)$, and defined as

$$
\text { Type } \mathcal{B R}(\alpha): \quad p_{m}^{(\alpha)}:=\frac{1}{\zeta(\alpha)} \frac{1}{m^{\alpha}}, \quad c(m)=m^{\alpha-1}, \quad \text { for } \alpha>1,
$$

and, of course, the Euclidean dynamical system with cost $c(m)=m$.

The following result describes the asymptotic behaviour of the Hausdorff dimension $s_{M}$ of the set $F_{M}$ relative to a Dirichlet boundary triple of the 
class $\mathcal{G} \mathcal{L G}$. It exhibits an exponential speed of convergence of $s_{M}$ towards 1 . When compared to (1.4), it proves that the constraints on each digit average are actually weaker than the constraints on each digit.

Theorem 2. Consider a Dirichlet boundary triple of $\mathcal{G} \mathcal{L G}$-type. Then the Hausdorff dimension of the set $F_{M}$ satisfies, as $M \rightarrow \infty$,

$$
\left|s_{M}-1\right|=\frac{C}{h} \exp \left[\frac{K}{C}-\gamma\right] e^{-M / C}\left[1+O\left(e^{-M \theta}\right)\right] \quad \text { with any } \theta<\frac{1}{C} .
$$

Here, $\gamma$ is the Euler constant, $h$ is the entropy, and $C, K$ are two constants relating to dominant spectral objects of the weighted transfer operator $\mathbf{H}_{s, w}$ defined in (1.7).

For the boundary Riemann triple $\mathcal{B R}(\alpha)$, for any $\theta<(\alpha-1) \zeta(\alpha)$ one has

$$
\left|s_{M}-1\right|=\frac{e^{\gamma(\alpha-2)}}{(\alpha-1) \zeta(\alpha) h(\alpha)} e^{-M(\alpha-1) \zeta(\alpha)}\left[1+O\left(e^{-M \theta}\right)\right]
$$

with

$$
h(\alpha)=\alpha \frac{\zeta^{\prime}(\alpha)}{\zeta(\alpha)}-\log \zeta(\alpha) .
$$

For the Euclidean dynamical system with $c(m)=m$, for any $\theta<2$ one has

$$
\left|s_{M}-1\right|=\frac{6}{\pi^{2}} e^{-1-\gamma} 2^{-M}\left[1+O\left(\theta^{-M}\right)\right]
$$

Relation to multifractal analysis. This work is partially related to multifractal analysis which was introduced by Mandelbrot for studying turbulence [34]. For a detailed survey of this question, see [14, 15]. For a dynamical system $(I, T)$, a fundamental interval relative to a finite prefix $\mathbf{m}:=\left(m_{1}, \ldots, m_{n}\right)$ is the interval of reals whose first $n$ digits form the prefix $\mathbf{m}$. For any $n$ and any $x$, the fundamental interval $I^{(n)}(x)$ is the interval of reals whose first $n$ digits are the same as the first $n$ digits of $x$. Each fundamental interval has two measures, the Lebesgue measure and another measure $\nu$ which is defined by the cost $c$. More precisely, for (normalized) $\operatorname{costs} c$ which give rise to a series $\sum_{m} \exp [-c(m)]=1$, the measure $\nu$ of a fundamental interval $I_{\mathbf{m}}$ related to the prefix $\mathbf{m}:=\left(m_{1}, \ldots, m_{n}\right)$ is defined by

$$
\left|\log \nu\left(I_{\mathbf{m}}\right)\right|=\sum_{i=1}^{n} c\left(m_{i}\right) .
$$

In this way, with respect to this measure $\nu$, the numeration process is memoryless and always produces the digit $m$ with probability $\exp [-c(m)]$. In order to compare the two measures, the Lebesgue measure and the measure $\nu$, multifractal analysis compares the two measures on fundamental 
intervals: it introduces the set $G_{\beta}$ of reals $x$ for which

$$
B_{n}(x):=\left|\frac{\log \nu\left(I^{(n)}(x)\right)}{\log \left|I^{(n)}(x)\right|}\right|=\left|\frac{C_{n}(x)}{\log \left|I^{(n)}(x)\right|}\right| \text { satisfies } \lim _{n \rightarrow \infty} B_{n}(x)=\beta,
$$

and studies the Hausdorff dimension $t_{\beta}$ of the set $G_{\beta}$.

For dynamical systems of the Good Class, the sequence $-n^{-1} \log \left|I^{(n)}(x)\right|$ tends almost everywhere to the entropy $h$, so that the asymptotic behaviour of the two sequences $M_{n}(x)$ and $h B_{n}(x)$ defined in (1.5) and (1.9) is the same almost everywhere. However, this is only true "almost everywhere". Finally, the relation between the two Hausdorff dimensions $s_{M}$ and $t_{\beta}$ is not so clear, and it is of great interest to compare our result on $F_{M}$ to the following result on $G_{\beta}$, recently obtained by Hanus, Mauldin and Urbański [18] which we translate in our setting.

TheOREM ([18]). Consider the set $G_{\beta}$ relative to a triple $(I, T, c)$ of $\mathcal{G M G}$-type. Suppose furthermore that the cost c satisfies $\sum_{m} \exp [-c(m)]=1$. Denote by $\mathbf{H}_{s, w}$ the weighted operator relative to the triple $(I, T, c)$ and by $\Lambda(s, w)$ the logarithm of its dominant eigenvalue when $\mathbf{H}_{s, w}$ acts on $\mathcal{C}^{1}(I)$. Then, for any $\beta$ near the value $\beta_{0}=\mu(c) / h$, there exists a unique pair $\left.(t, w)=\left(t_{\beta}, w_{\beta}\right) \in[0,1] \times\right]-\infty,+\infty[$ for which

$(\mathcal{G}): \quad \Lambda(t-\beta w, w)=0, \quad \frac{\partial}{\partial w} \Lambda(t-\beta w, w)=-\beta \frac{\partial}{\partial s} \Lambda(t-\beta w, w)$.

The Hausdorff dimension of $G_{\beta}$ equals $t_{\beta}$. The two functions $\beta \mapsto t_{\beta}$, $\beta \mapsto w_{\beta}$ are analytic for $\beta$ near $\beta_{0}$.

Note that even if the two results (our Theorem 1 and the previous Theorem) are of the same spirit and involve the same kind of systems $(\mathcal{F})$ and $(\mathcal{G})$, the result on $G_{\beta}$ is obtained in the $\mathcal{G} \mathcal{M G}$-setting, while ours is obtained in the $\mathcal{G} \mathcal{L} \mathcal{G}$-setting. This explains why the methods used cannot be similar. They both deal with the weighted transfer operator $\mathbf{H}_{s, w}$; however, the authors in [18] used analyticity of $(s, w) \mapsto \mathbf{H}_{s, w}$ at $(1,0)$, together with ergodic theorems; this is not possible here, and we have to introduce other tools, similar to those used in large deviations results.

Plan of the paper. In Section 2, we introduce the main tools, and we describe the $\mathcal{G} \mathcal{L} \mathcal{G}$-setting. Section 3 proves that the Hausdorff dimension of $F_{M}$ can be described only in terms of fundamental intervals (Proposition 1). Section 4 recalls the main properties of the weighted transfer operator (Proposition 2) and its spectral objects, mainly its dominant eigenvalue $\lambda(s, w)$. Section 5 relates the Hausdorff dimension to $\lambda(s, w)$ in Proposition 3 and proves Theorem 1. Section 6 is devoted to introducing the Dirichlet boundary triples and proving Theorem 2 .

Some of these results have been presented at the Colloquium on Mathematics and Computer Science: Algorithms, Trees, Combinatorics and Prob- 
ability (Vienna, September 2004). An extended abstract can be found in the proceedings of this conference [10]. However, our Theorem 2 is much more general than the corresponding theorem in [10], and most of the results in Section 6 are new.

\section{DYNAMICAL SYSTEMS, NUMERATION SCHEMES, AND WEIGHTS}

We first recall some general definitions about dynamical systems, numeration schemes and costs (or weights) related to trajectories. We then introduce the set $F_{M}$.

2.1. Numeration schemes. We first define a subclass of dynamical systems (on the interval) well-adapted to our purposes. For a readable treatment of dynamical systems on the interval, see [32].

Definition 1. A dynamical system of the Good Class is defined by four elements:

(i) An alphabet $\mathcal{M}$ included in $\mathbb{N}^{\star}$, whose elements are called digits.

(ii) A topological partition of $I:=[0,1]$ into disjoint open intervals $I_{m}$, $m \in \mathcal{M}$, i.e., $[0,1]=\bigcup_{m \in \mathcal{M}} \bar{I}_{m}$; the length of the interval $I_{m}$ is denoted by $p_{m}$.

(iii) A mapping $\varrho$ which is constant and equal to $m$ on each $I_{m}$.

(iv) A mapping $T$, often called the shift, whose restriction to each $\bar{I}_{m}$ is a $\mathcal{C}^{2}$ bijection from $\bar{I}_{m}$ to $I$. Let $h_{m}$ be the inverse branch of $T$ restricted to $\bar{I}_{m}$. The mappings $h_{m}$ satisfy the following:

(a) [Contraction] For each $m \in \mathcal{M}$, there exist $\eta_{m}, \delta_{m}$ with $0<$ $\eta_{m}<\delta_{m}<1$ for which $\eta_{m} \leq\left|h_{m}^{\prime}(x)\right| \leq \delta_{m}$ for $x \in I$. The quantity $\delta:=\sup _{m \in \mathcal{M}} \delta_{m}$ satisfies $\delta<1$ and is called the contraction ratio.

(b) [Bounded distortion property] There exists a constant $r>0$, called the distortion constant, such that $\left|h_{m}^{\prime \prime}(x)\right| \leq r\left|h_{m}^{\prime}(x)\right|$ for all $m \in \mathcal{M}$ and for all $x \in I$.

(c) [Convergence on the left of $s=1$ ] There exists $\sigma<1$ for which the series $\sum_{m \in \mathcal{M}} p_{m}^{\sigma}$ is convergent. The infimum $\sigma_{0}$ of such $\sigma$ is the abscissa of convergence.

With a system of the Good Class, a representation scheme for real numbers of $I$ is built as follows: We relate to $x$ its trajectory

$$
\mathcal{T}(x)=\left(x, T(x), T^{2}(x), \ldots\right) \text {. }
$$

As soon as $x$ is ordinary, i.e., it does not belong to the exceptional set $\mathcal{E}:=$ $\bigcup_{n \geq 0} T^{-n}(\{0,1\})$, this trajectory can be encoded by the unique (infinite) sequence of digits produced by applying the map $\varrho$ to each element $T^{i}(x)$ 
of the trajectory:

$$
\left(m_{1}(x), m_{2}(x), \ldots\right) \quad \text { with } \quad m_{i}(x):=\varrho\left(T^{i-1}(x)\right) .
$$

Each branch (or inverse branch) of the $n$th iterate $T^{n}$ of the shift $T$ is called a branch of depth $n$. It is then associated in a unique way to an $n$-uple $\mathbf{m}=\left(m_{1}, \ldots, m_{n}\right)$ of length $n$, and is of the form $h_{\mathbf{m}}:=h_{m_{1}} \circ \cdots \circ h_{m_{n}}$. The set of inverse branches of depth $n$ is exactly $\mathcal{H}^{n}$, and the set of all the inverse branches of any depth is $\mathcal{H}^{\star}$. Together, the distortion and contraction properties entail the existence of a constant $L>0$ such that

$$
\frac{1}{L} \leq\left|\frac{h^{\prime}(x)}{h^{\prime}(y)}\right| \leq L \quad \text { for any } h \in \mathcal{H}^{\star} .
$$

Definition 2. For any $n$-uple of digits, of the form $\mathbf{m}=\left(m_{1}, \ldots, m_{n}\right)$, the interval $I_{\mathbf{m}}:=h_{\mathbf{m}}(] 0,1[)$ gathers all the reals $x$ for which the sequence of the first $n$ digits equals $\mathbf{m}$; it is called the fundamental interval relative to $\mathbf{m}$. Its depth equals the length $|\mathbf{m}|$ of the prefix $\mathbf{m}$ and its Lebesgue measure denoted by $p_{\mathbf{m}}$ satisfies $p_{\mathbf{m}} \leq \delta^{n}$.

2.2. Main examples. Here we focus on dynamical systems relative to an infinite alphabet $\mathcal{M}$. The most classical examples are memoryless sources (of Riemann type) and the continued fraction expansion.

Continued fraction expansion. The shift $T$, also known as the Gauss map, is

$$
T(x)=\frac{1}{x}-\left\lfloor\frac{1}{x}\right\rfloor \text { for } x \neq 0, \quad T(0)=0 .
$$

It is relative to the topological partition $I_{m}=(1 /(m+1), 1 / m)$. The inverse branch of depth 1 associated to the digit $m$ is the LFT (linear fractional transformation) $h_{m}(z)=1 /(m+z)$. This map induces the numeration scheme related to continued fraction expansion.

Memoryless dynamical systems. A dynamical system of the Good Class is memoryless when the branches $h_{m}$ are affine. It is completely defined (up to isomorphism) by the length $p_{m}=\delta_{m}$ of each interval $I_{m}$ of depth one, which equals the probability $p_{m}$ of emitting $m$ at each step of the process. The fundamental interval can be chosen as $\left.I_{m}:=\right] q_{m}, q_{m+1}$ [ with $q_{1}=0$ and $q_{m}:=\sum_{k<m} p_{k}$. A special type of memoryless source is studied here as a main example: the Riemann type $\mathcal{R}(\alpha)$ (for $\alpha>1$ ) where the associated probabilities are

$$
p_{m}^{(\alpha)}:=\frac{1}{\zeta(\alpha)} \frac{1}{m^{\alpha}} .
$$

The affine approximation of the Gauss map is the memoryless system rela- 
tive to the partition $I_{m}=(1 /(m+1), 1 / m)$. The length $p_{m}$ is $1 /(m(m+1))$, and it is of the same type as the system $\mathcal{R}(2)$.

2.3. Costs and weighted prefix averages. A digit-cost $c$ relative to a dynamical system $(I, T)$ is a strictly positive function $c: \mathcal{M} \rightarrow \mathbb{R}^{+}$which extends to a function $c: \mathcal{M}^{\star} \rightarrow \mathbb{R}^{+}$via the additive property

$$
c(\mathbf{m}):=\sum_{i=1}^{n} c\left(m_{i}\right) \quad \text { for } \mathbf{m}=\left(m_{1}, \ldots, m_{n}\right) .
$$

On each trajectory $\mathcal{T}(x)$ of an ordinary $x$, encoded by the sequence $\left(m_{1}(x)\right.$, $\left.m_{2}(x), \ldots\right)$ defined in $(1.1)$, we recall that the weighted prefix average of length $n$ is defined as

$$
M_{n}(x):=\frac{1}{n} C_{n}(x) \quad \text { with } \quad C_{n}(x):=\sum_{i=1}^{n} c\left(m_{i}(x)\right),
$$

and we study here the set $F_{M}$ of reals for which all the $M_{n}(x)$ are bounded by $M$.

2.4. Triples of large growth. The strength of these constraints depends on the relation between the cost $c$ and the occurrence probability of the digits. Consider the (initial) probability distribution $p: k \mapsto p_{k}:=$ $\mathbb{P}\left[m_{1}=k\right]$ of digit $m_{1}$, together with the limit distribution $\bar{p}$ of the $n$th digit $m_{n}$ which always exists in the Good Class setting. We are mostly interested in the case of a dynamical system of the Good Class, with an infinite alphabet $\mathcal{M}$, where the sequences $p_{m}, c(m)$ satisfy

(c1) $m \mapsto p_{m}$ is decreasing,

(c2) $m \mapsto c(m)$ is strictly increasing to $+\infty$.

The mixed sequences $\pi_{n}:=\min \left\{p_{m} ; c(m) \leq n\right\}, \bar{\pi}_{n}:=\min \left\{\bar{p}_{m} ; c(m) \leq n\right\}$ summarize the balance between the increase of the cost $c$ and the decrease of the distributions $p, \bar{p}$, and the conditions

(c3) $\liminf \pi_{n}^{1 / n}=1$ and $\liminf \bar{\pi}_{n}^{1 / n}=1$

(which are equivalent for systems of the Good Class) informally express that the increase of $c$ is faster than the decrease of $p$. Since $\pi_{n} \leq 1$, condition (c3) implies $\lim \sup \pi_{n}^{1 / n}=1$ and this last condition is equivalent to requiring that the convergence radii of the series $U(z), V(z)$ defined as

$$
U(z):=\sum_{n \geq 1} \pi_{n} z^{n}, \quad V(z):=\sum_{m \geq 1} \bar{p}_{m} z^{\lfloor c(m)\rfloor}
$$


equal 1. Furthermore, consider the series

$$
P(s, u):=\sum_{m=1}^{\infty} \frac{1}{c(m)^{u}} \bar{p}_{m}^{s} .
$$

Recalling that $\sigma_{0}$ is the convergence abscissa of $P(s, 0)$ (see Def. 1(iv)(c)), and defining $\sigma_{1}$ as the convergence abscissa of $P(s,-1)$ (we call it the critical abscissa), we also require

(c4) $P\left(\sigma_{0}, 0\right)=\infty, P\left(\sigma_{1},-1\right)=\infty$.

This leads us to the following definition:

Definition 3. Consider a triple $(I, T, c)$ with a system $(I, T)$ of the Good Class. If it does not satisfy condition (c3), it is said to be of moderate growth $(\mathcal{G M G}$-setting). If it satisfies conditions $(\mathrm{c} 1)-(\mathrm{c} 4)$, it is said to be of large growth $\left(\mathcal{G} \mathcal{L G}\right.$-setting). Finally, when the first critical abscissa $\sigma_{1}$ equals 1 , the triple $(I, T, c)$ is called a boundary triple.

Here, we focus on the $\mathcal{G} \mathcal{L} \mathcal{G}$-setting. In the boundary case, the (stationary) average $\mu(c)$ of the cost $c$ defined in (1.6) is infinite.

Examples of triples $(I, T, c)$ of $\mathcal{G} \mathcal{L G}$-type. We mainly consider instances where $c(m)=\Theta\left(p_{m}^{-d}\right)$ with some real $d>0$. Then the abscissa $\sigma_{1}$ equals $\sigma_{0}+d$. There is a special type of triples related to memoryless sources that is studied here as a main example: the Riemann triples $\mathcal{R}(\alpha, \beta)$ (for $\alpha>1$ and $\beta \geq \alpha-1$ ),

$$
\text { Type } \mathcal{R}(\alpha, \beta): \quad p_{m}^{(\alpha)}:=\frac{1}{\zeta(\alpha)} \frac{1}{m^{\alpha}}, \quad c(m)=m^{\beta}, \quad \text { for } \quad \beta>0 .
$$

The triple $\mathcal{R}(\alpha, \beta)$ is boundary if $\beta=\alpha-1$, and is then denoted by $\mathcal{B R}(\alpha):=$ $\mathcal{R}(\alpha, \alpha-1)$.

An important triple of $\mathcal{G} \mathcal{L G}$-type is formed by the Gauss map $(I, T)$ together with the cost $c(m)=m^{\beta}$ with $\beta>0$. When $\beta=1$, one obtains a boundary triple which will be one of the most interesting examples in our study. Note that $\mathcal{B R}(2)$ provides an approximate memoryless version of this triple.

2.5. Subsets $F_{M}$. Here, we wish to study the sets $F_{M}$ that are associated to a triple $(I, T, c)$ of class $\mathcal{G} \mathcal{L G}$ as follows.

Definition 4. Consider a triple $(I, T, c)$ of $\mathcal{G} \mathcal{L G}$-type. For any $M>$ $\gamma(c)$, the set $F_{M}$ is the set of ordinary reals $x$ of $I$ for which all the weighted averages $M_{n}(x)$ defined in (1.5) satisfy $M_{n}(x) \leq M$. The set $\widetilde{F}_{M}$ is the set of ordinary reals $x$ of $I$ for which the sequence $M_{n}(x)$ satisfies $M_{n}(x) \leq M$ for $n$ sufficiently large. 
For any $M \in] \gamma(c), \mu(c)\left[\right.$, the Lebesgue measures of the sets $F_{M}$ and $\widetilde{F}_{M}$ equal 0 , and we wish to study their Hausdorff dimensions. The next result shows that they are the same.

Lemma 1. The sets $F_{M}$ and $\widetilde{F}_{M}$ have the same Hausdorff dimension.

Proof. Note that $\widetilde{F}_{M}$ is the (disjoint) union of the sets $F_{M}^{(k)}$ where $F_{M}^{(k)}=\left\{x \in \widetilde{F}_{M} ; k\right.$ is the smallest integer such that $\left.C_{n}(x) \leq M n, \forall n \geq k\right\}$. Note also that if $x \in F_{M}^{(k)}$, then $C_{k-1}(x)>M(k-1)$ whereas $C_{n}(x) \leq M n$ for all $n \geq k$. Thus, for all $n \geq k$, the relation

$$
C_{n}(x)-C_{k-1}(x)=\sum_{m=k}^{n} c\left(m_{i}(x)\right)=C_{n-k+1}\left[T^{k-1} x\right],
$$

together with the bound

$$
\sum_{m=k}^{n} c\left(m_{i}(x)\right) \leq M n-M(k-1)=M(n-k+1),
$$

proves the inequality $C_{n-k+1}\left[T^{k-1} x\right] \leq M(n-k+1)$. This implies that if $x \in F_{M}^{(k)}$, then $T^{k-1}(x) \in F_{M}$. Finally,

$$
F_{M}^{(k+1)} \subset T^{-k}\left(F_{M}\right), \quad \widetilde{F}_{M} \subset \bigcup_{h \in \mathcal{H}^{\star}} h\left(F_{M}\right) .
$$

The set $\mathcal{H}^{\star}$ is denumerable, as the denumerable union of denumerable sets. Condition (iv)(a) of Definition 1 entails that each element $h$ of $\mathcal{H}^{\star}$ is bi-Lipschitz, so that $h\left(F_{M}\right)$ and $F_{M}$ have the same Hausdorff dimension, and finally $\operatorname{dim} \widetilde{F}_{M} \leq \operatorname{dim} F_{M}$. Since the reverse inequality is clear, the proof is complete.

2.6. Covers of the set $F_{M}$. For each $n$, we consider the two subsets of $\mathcal{M}^{n}$,

$$
\begin{gathered}
\mathcal{A}_{n}(M):=\left\{\mathbf{m} \in \mathcal{M}^{n} ; c(\mathbf{m}) \leq M n\right\}, \\
\mathcal{B}_{n}(M):=\left\{\mathbf{m} \in \mathcal{M}^{n} ; \forall r \leq n \mathbf{m}_{\mid r}:=\left(m_{1}, \ldots, m_{r}\right) \in \mathcal{A}_{r}(M)\right\},
\end{gathered}
$$

and the two subsets of $I$,

$$
A_{n}(M):=\bigcup_{\mathbf{m} \in \mathcal{A}_{n}(M)} I_{\mathbf{m}}, \quad B_{n}(M):=\bigcup_{\mathbf{m} \in \mathcal{B}_{n}(M)} I_{\mathbf{m}} .
$$

We remark that the set $F_{M}$ can be defined in two ways, with the $A_{n}(M)$ sequence or the $B_{n}(M)$ sequence:

$$
F_{M}=\bigcap_{n \geq 1} A_{n}(M), \quad F_{M}=\bigcap_{n \geq 1} B_{n}(M) .
$$


The sequence $B_{n}(M)$ has good properties from the point of view of covers, whereas $A_{n}(M)$ gives rise to good properties of operators. There is a close link between these two sequences, due to the next lemma. This lemma is of the same spirit as the so-called "cyclic lemma", which is useful in the random walk setting.

LEMMA 2. For any $\mathbf{m} \in \mathcal{A}_{n}(M)$, there exists a circular permutation $\tau$ for which $\tau(\mathbf{m}) \in \mathcal{B}_{n}(M)$.

Proof. For $\mathbf{m} \in \mathcal{A}_{n}(M)$, denote by $r_{1}$ the maximum value of $r$ such that $\sum_{i=1}^{r} c\left(m_{i}\right)>M r$. If $r_{1}$ does not exist, then $\tau(\mathbf{m})=\mathbf{m}$. Otherwise, $r_{1}<n$, and the block formed with the last $n-r_{1}$ digits is well behaved, since

$$
\sum_{i=r_{1}+1}^{r} c\left(m_{i}\right)<M\left(r-r_{1}\right) \quad \text { for all } r \text { such that } r_{1}<r \leq n \text {. }
$$

This is due to the inequalities

$$
\begin{aligned}
M r & \geq\left(r-r_{1}\right) \frac{\sum_{i=r_{1}+1}^{r} c\left(m_{i}\right)}{r-r_{1}}+r_{1} \frac{\sum_{i=1}^{r_{1}} c\left(m_{i}\right)}{r_{1}} \\
& >\left(r-r_{1}\right) \frac{\sum_{i=r_{1}+1}^{r} c\left(m_{i}\right)}{r-r_{1}}+r_{1} M .
\end{aligned}
$$

Since the block formed with the last $n-r_{1}$ digits is well-behaved, we wish to place it at the beginning of the sequence $\mathbf{m}$. We thus consider a circular permutation $\tau$ defined by $\tau(\mathbf{m})=\mathbf{u}$ with

$$
u_{i}:=m_{r_{1}+i} \quad\left(1 \leq i \leq n-r_{1}\right), \quad u_{i}:=m_{i-n+r_{1}} \quad\left(n-r_{1}<i \leq n\right) .
$$

By $(2.9)$, the average of $c\left(u_{1}\right), \ldots, c\left(u_{s}\right)$ is at most $M$ for $s$ varying between 1 and $n-r_{1}$.

Now, consider the maximum value $r_{2}$ of $r$ such that $\sum_{i=1}^{r} c\left(u_{i}\right)>M r$. If $r_{2}$ does not exist, we can stop here and the sequence $\tau(\mathbf{m}):=\left(u_{1}, \ldots, u_{n}\right)$ belongs to $\mathcal{B}_{n}(M)$. Otherwise, $n-r_{1}<r_{2}<n$. By repeating the same construction as before we obtain a new string $\left(a_{1}, \ldots, a_{n}\right)$, but the average of the first $n-r_{1}+n-r_{2}$ digits is at most $M$.

Proceeding in this way, it is possible to get a sequence of strictly positive integers $n-r_{1}, n-r_{2}, \ldots$ whose sum cannot exceed $n$. Hence, this procedure must stop after a finite number of steps, and yields the circular permutation $\tau$.

\section{HAUSDORFF DIMENSION OF SETS CONSTRAINED BY THEIR PREFIXES}

We first recall some classical facts about covers and Hausdorff dimension. The definition of Hausdorff dimension of a given set a priori involves all its possible covers. Here, we introduce a class of sets (the sets which are 
well-constrained by their prefixes) which contains all the sets $F_{M}$ relative to triples of large growth. We prove in Proposition 1 that, for such sets, the Hausdorff dimension can be determined via particular covers, formed with fundamental intervals of fixed depth. For sets $F_{M}$, this characterization involves the sets $\mathcal{B}_{n}(M)$ of (2.7).

3.1. Covers and Hausdorff dimension. Let $E \subset I$. A cover $\mathcal{J}:=$ $\left(J_{\ell}\right)_{\ell \in \mathcal{L}}$ of $E$ is a set of open intervals $J_{\ell}$ for which $E \subset \bigcup_{\ell \in \mathcal{L}} J_{\ell}$. It is said to be finite if $\mathcal{L}$ is finite. The diameter of a cover is the real $\varrho$ that is the supremum of the lengths $\left|J_{\ell}\right|$. A cover is fundamental [with respect to some dynamical system $(I, T)$ ] if its elements $J_{\ell}$ are fundamental intervals. For each cover $\mathcal{J}$ of $E$, the quantity

$$
\Gamma_{\sigma}(\mathcal{J}):=\sum_{J \in \mathcal{J}}|J|^{\sigma}
$$

plays a fundamental rôle in the following.

A subset $E$ of $I$ is said to have zero measure in dimension $\sigma$ [we write $\left.\mu_{\sigma}(E)=0\right]\left({ }^{1}\right)$ if for any $\varepsilon>0$, there exists a cover $\mathcal{K}$ of $E$ for which $\Gamma_{\sigma}(\mathcal{K})<\varepsilon$. A subset $E$ of $I$ is said to have an infinite measure in dimension $\sigma$ [written $\mu_{\sigma}(E)=\infty$ ] if for any $A>0$, there exists $\varrho>0$ such that $\Gamma_{\sigma}(\mathcal{K})>A$ for any cover $\mathcal{K}$ of $E$ of diameter at most $\varrho$.

The Hausdorff dimension of $E$, denoted by $\operatorname{dim} E$, is the unique number $d$ for which $\mu_{\sigma}(E)=0$ for any $\sigma>d$ and $\mu_{\sigma}(E)=+\infty$ for any $\sigma<d$ :

$$
\operatorname{dim} E=\inf \left\{\sigma ; \mu_{\sigma}(E)=0\right\}=\sup \left\{\sigma ; \mu_{\sigma}(E)=+\infty\right\} .
$$

This definition of the Hausdorff dimension involves all possible covers of the set $E$. We show in this section that it is sufficient to deal with particular covers consisting of fundamental intervals of the same depth. In the multifractal analysis framework, studies of the Hausdorff dimension of the sets $G_{\beta}$ defined in (1.9) have already been performed, but only in the $\mathcal{G M G}$-setting. When the dynamical system has a finite number of branches, it is proven (see for instance $[37,38]$ ) that it is sufficient to deal with covers consisting of fundamental intervals of the same depth. The fact that the number of branches is finite plays an important rôle there, since one deals with the quantity $\eta:=\inf \eta_{m}$ of Definition 1(iv)(a) and mainly uses the fact that $\eta$ is strictly positive. Always in the $\mathcal{G} \mathcal{M G}$-setting, now when the dynamical system has an infinite number of branches, as in [18], previous analyses deal with a set called the Bad Set and devote a considerable amount of work to proving that the Bad Set does not actually intervene in the computation of the Hausdorff dimension.

$\left({ }^{1}\right)$ Here, $\mu_{\sigma}$ does not denote a measure, we just use it as a notation. 
In this section, we shall prove directly that it is sufficient to deal with covers formed by fundamental intervals of the same depth for studying the Hausdorff dimension of the sets $F_{M}$, even if the dynamical system has an infinite number of branches.

3.2. Sets which are well-constrained by their prefixes. We are interested in studying sets of the same type as $F_{M}$, and we will consider in this section a more general class of sets which are defined by constraints on their prefixes of any length.

Definition 5. Let $(I, T)$ be a dynamical system of the Good Class, and $\mathcal{M}$ its associated alphabet. A subset $E$ is defined by its prefixes if there exists a sequence $\mathcal{M}_{\star}:=\left(\mathcal{M}_{n}\right)_{n \geq 1}$ of non-empty subsets $\mathcal{M}_{n} \subset \mathcal{M}^{n}$ (the constraints) for which

$$
E:=\bigcap_{n \geq 1} \bigcup_{\mathbf{m} \in \mathcal{M}_{n}} I_{\mathbf{m}}
$$

The sequence $\mathcal{M}_{\star}$ is the canonical sequence of $E$. Moreover, if $\mathcal{M}_{\star}$ satisfies the following four conditions:

(i) for any $n \geq 1$, the set $\mathcal{M}_{n}$ is finite,

(ii) if $\left(m_{1}, \ldots, m_{n}\right) \in \mathcal{M}_{n}$ then $\left(m_{1}, \ldots, m_{n-1}\right) \in \mathcal{M}_{n-1}$,

(iii) $\mathcal{M}_{n_{1}} \times \mathcal{M}_{n_{2}} \subset \mathcal{M}_{n_{1}+n_{2}}$ for all $n_{1}, n_{2}$,

(iv) the sequence defined as $\pi_{n}:=\min \left\{p_{m_{n}} ; \exists\left(m_{1}, \ldots, m_{n-1}\right) \in \mathcal{M}^{n-1}\right.$ such that $\left.\left(m_{1}, \ldots, m_{n}\right) \in \mathcal{M}_{n}\right\}$ satisfies $\liminf \pi_{n}^{1 / n}=1$,

then the sequence $\mathcal{M}_{\star}$ is said to be well-conditioned. In this case, the set $E$ is said to be well-constrained by its prefixes (briefly, a $\mathcal{W C P}$ set). For each $n$, the set $\mathcal{J}_{n}:=\left\{I_{\mathbf{m}} ; \mathbf{m} \in \mathcal{M}_{n}\right\}$ is a cover of $E$, which is finite and fundamental. The sequence $\left(\mathcal{J}_{n}\right)$ is called the canonical system of covers of $E$.

Lemma 3. Consider a triple $(I, T, c)$ of $\mathcal{G} \mathcal{L G}$-type. For $M>\gamma(c)$, the sequence $\mathcal{B}_{n}(M)$ defined in $(2.7)$ is well-conditioned and the set $F_{M}$ is wellconstrained by its prefixes.

Proof. (i) is true since $c$ is strictly increasing to $+\infty$. (ii) is due to the definition of $\mathcal{B}_{n}(M)$, while (iii) comes from the additivity of the cost. (iv) is just the condition (c3) of Section 2.4 on the triple $(I, T, c)$.

3.3. Hausdorff dimension of $\mathcal{W C P}$ sets. The following proposition shows that the Hausdorff dimension of a set $E$ which is well-constrained by its prefixes can be uniquely characterized via its canonical system of covers $\left(\mathcal{J}_{n}\right)$ and the quantities $\Gamma_{\sigma}$ defined in (3.1).

Proposition 1 [Characterization of the Hausdorff dimension of a $\mathcal{W C P}$ set via its canonical system of covers]. Let $E$ be a subset of $I$ which is 
well-constrained by its prefixes, and $\left(\mathcal{J}_{n}\right)_{n \geq 1}$ its canonical system of covers. Then

$$
\operatorname{dim} E=\inf \left\{\sigma ; \sup \left\{\Gamma_{\sigma}\left(\mathcal{J}_{n}\right) ; n \geq 1\right\}<\infty\right\}
$$

Proof. It is based on four lemmata. The upper bound for $\operatorname{dim} E$ is easy to obtain (Lemma 4). The proof of the converse inequality is more involved and uses Lemmata 5-7. The proof of Lemma 5 mainly uses condition (iv) of Definition 5 which is equivalent to a condition introduced by J. Peyrière [39]. Finally, Lemma 6 provides a characterization of the Hausdorff dimension which involves covers formed by fundamental intervals of variable depth. Then Lemma 7 (which extends Lemma 7 of [17]) shows that the Hausdorff dimension is completely characterized by covers of fixed depth.

Lemma 4. Assume that the hypotheses of Proposition 1 hold. Then

$$
\operatorname{dim} E \leq \inf \left\{\sigma ; \sup \left\{\Gamma_{\sigma}\left(\mathcal{J}_{n}\right) ; n \geq 1\right\}<\infty\right\}
$$

Proof. Suppose that $\sup \left\{\Gamma_{\sigma}\left(\mathcal{J}_{n}\right) ; n \geq 1\right\}<\infty$ and consider some $\sigma^{\prime}>\sigma$. Since the contraction property entails that $p_{\mathbf{m}}^{\sigma^{\prime}} \leq p_{\mathbf{m}}^{\sigma} \cdot \delta^{n\left(\sigma^{\prime}-\sigma\right)}$ for each $\mathbf{m} \in \mathcal{M}^{n}$, one has

$$
\Gamma_{\sigma^{\prime}}\left(\mathcal{J}_{n}\right) \leq \Gamma_{\sigma}\left(\mathcal{J}_{n}\right) \delta^{n\left(\sigma^{\prime}-\sigma\right)},
$$

and $\Gamma_{\sigma^{\prime}}\left(\mathcal{J}_{n}\right)$ tends to 0 . Hence, by definition of the Hausdorff dimension $d$ of $E$, one has $d \leq \sigma^{\prime}$.

Lemma 5. Assume that the hypotheses of Proposition 1 hold. For any $\varrho>0$, denote by $\mathcal{J}_{\star}$ the union of $\mathcal{J}_{n}$ and by $\mathcal{J}_{\star}^{(\varrho)}$ the set of intervals $J$ of $\mathcal{J}_{\star}$ for which $|J| \leq \varrho$. Consider any finite cover $\mathcal{K}$ of $E$ with $\mathcal{K} \subset \mathcal{J}_{\star}^{(\varrho)}$, and define

$$
\Gamma_{\sigma}^{(\varrho)}(E)=\inf \left\{\Gamma_{\sigma}(\mathcal{K}) ; \mathcal{K} \in \mathcal{J}_{\star}^{(\varrho)}, \mathcal{K} \text { covers } E\right\}, \quad \underline{\Gamma}_{\sigma}(E)=\lim _{\varrho \rightarrow 0} \Gamma_{\sigma}^{(\varrho)}(E) .
$$

Then $\operatorname{dim} E=\inf \left\{\sigma ; \underline{\Gamma}_{\sigma}(E)=0\right\}=\sup \{\sigma ; \underline{\Gamma} \sigma(E)=\infty\}$.

Proof. This result is an adaptation of a result due to J. Peyrière who uses, for any $J \in \mathcal{J}_{\star}$, the quantity

$$
\tau(J):=\sup \left\{|J| /|K| ; K \subset J, K \in \mathcal{J}_{\star}, d(K)=d(J)+1\right\},
$$

where $d(\cdot)$ denotes the depth of a fundamental interval. He shows that the condition (called here the Peyrière condition)

$$
\forall \alpha>0, \quad \lim \sup \left\{\tau(J)|J|^{\alpha} ; J \in \mathcal{J}_{\star},|J| \rightarrow 0\right\} \leq 1
$$

is sufficient to imply the conclusion of this lemma. Here, condition (iv) of Definition 5 implies the Peyrière condition: together with the distortion and contraction properties (Definition 1(iv)(a), (b)), it entails

$$
\tau(J) \leq \frac{1}{L \pi_{n+1}} \quad \text { and } \quad|J|^{\alpha} \leq \delta^{n \alpha} \quad \text { if } d(J)=n
$$


For a given cover $\mathcal{K}$, define the lower depth of $\mathcal{K}$ as $\min \{d(J): J \in \mathcal{K}\}$ where $d(\cdot)$ denotes the depth of a fundamental interval. The next lemma gives a lower bound for the Hausdorff dimension by considering covers of sufficiently large lower depth.

Lemma 6. Assume that the hypotheses of Proposition 1 hold and there exists a natural number $n_{0}$ such that, for any finite cover $\mathcal{K}$ of $E$ which is a subcover of $\mathcal{J}_{\star}$ of lower depth greater than $n_{0}$, one has $\Gamma_{\sigma}(\mathcal{K})>1$. Then $\operatorname{dim} E \geq \sigma$.

Proof. Suppose that there exists such an $n_{0}$ and consider $\varrho>0$ such that

$$
\varrho \leq \varrho_{0}:=\min \left\{p_{\mathbf{m}} ; \mathbf{m} \in \mathcal{M}_{n}, n<n_{0}\right\} .
$$

[Since all the $\mathcal{M}_{n}$ are finite, this minimum $\varrho_{0}$ exists and is strictly positive.] Consider now a finite cover $\mathcal{K}$ of $E$ which is a subcover of $\mathcal{J}^{(\varrho)}$. Its lower depth is at least $n_{0}$, and so $\Gamma_{\sigma}(\mathcal{K}) \geq 1$. Finally, $\Gamma_{\sigma}^{(\varrho)}(E) \geq 1$ for all $\varrho<\varrho_{0}$. Then $\underline{\Gamma}_{\sigma}(E) \geq 1$, and therefore, by Lemma $5, \sigma$ must be smaller than $\operatorname{dim} E$.

Lemma 7. Assume that the hypotheses of Proposition 1 hold. Then

$$
\operatorname{dim} E \geq \inf \left\{\sigma ; \sup \left\{\Gamma_{\sigma}\left(\mathcal{J}_{n}\right) ; n \in \mathbb{N}\right\}<\infty\right\} .
$$

Proof. Consider $\sigma$ for which $\sup \left\{\Gamma_{\sigma}\left(\mathcal{J}_{n}\right) ; n \in \mathbb{N}\right\}$ equals $\infty$. Then there exists an index $n_{1}$ for which $\Gamma_{\sigma}\left(\mathcal{J}_{n_{1}}\right)>2 L^{2 \sigma}$.

STEP 1. We show that if $n$ is large enough, then $\Gamma_{\sigma}\left(\mathcal{J}_{n}\right)>1$.

First note that, for fixed $n_{1}$ and any $n$, the distortion property (2.1) and hypothesis (ii) of Definition 5 together entail that

$$
\Gamma_{\sigma}\left(\mathcal{J}_{n_{1}+n}\right) \geq L^{-2 \sigma} \Gamma_{\sigma}\left(\mathcal{J}_{n_{1}}\right) \Gamma_{\sigma}\left(\mathcal{J}_{n}\right) \geq L^{-2 \sigma} 2 L^{2 \sigma} \Gamma_{\sigma}\left(\mathcal{J}_{n}\right) \geq 2 \Gamma_{\sigma}\left(\mathcal{J}_{n}\right) .
$$

An inductive argument shows that, for $t \in \mathbb{N}$, one has $\Gamma_{\sigma}\left(\mathcal{J}_{t n_{1}}\right) \geq 2^{t-1} \Gamma_{\sigma}\left(\mathcal{J}_{n_{1}}\right)$ $\geq 2^{t}$. Now, any $n \geq n_{1}$ can be written as $n=t n_{1}+r$ with $0 \leq r<n_{1}$, and

$$
\Gamma_{\sigma}\left(\mathcal{J}_{n}\right)=\Gamma_{\sigma}\left(\mathcal{J}_{r+t n_{1}}\right) \geq L^{-2 \sigma} \Gamma_{\sigma}\left(\mathcal{J}_{t n_{1}}\right) \Gamma_{\sigma}\left(\mathcal{J}_{r}\right) \geq 2^{t} L^{-2 \sigma} \Gamma_{\sigma}\left(\mathcal{J}_{r}\right) .
$$

Denote by $\alpha(n)$ the maximum of $\left\{\left|I_{\mathbf{m}}\right|^{-1} ; \mathbf{m} \in \mathcal{M}_{n}\right\}$. The quantity $\alpha(n)$ is well defined since $\mathcal{M}_{n}$ is finite. Consider an integer $t_{0} \geq 1$ for which $2^{t_{0}}>L^{2 \sigma} \alpha\left(n_{1}\right)$. Then $\left|I_{\mathbf{m}}\right|^{\sigma} \geq \alpha(r)^{-\sigma}$ for any $\mathbf{m} \in \mathcal{M}_{r}$, and

$$
\Gamma_{\sigma}\left(\mathcal{J}_{r}\right)=\sum_{\mathbf{m} \in \mathcal{M}_{r}}\left|I_{\mathbf{m}}\right|^{\sigma} \geq \alpha(r)^{-\sigma}>\alpha\left(n_{1}\right)^{-\sigma}>\alpha\left(n_{1}\right)^{-1} .
$$

Finally, by the definition of $t_{0}$, the inequality $\Gamma_{\sigma}\left(\mathcal{J}_{n}\right)>1$ holds for any $n \geq n_{2}:=t_{0} n_{1}$, which ends the first step.

SteP 2. Consider now a multiple $n_{3}$ of $n_{1}$ greater than $n_{2}$. Denote by $\mathcal{K}$ any subcover of $\mathcal{J}_{\star}$ formed by intervals $J$ with depth a multiple of $n_{3}$. We will show that $\Gamma_{\sigma}(\mathcal{K})>L^{-2 \sigma}$. 
Suppose first that $\mathcal{K}$ contains intervals of depth $n_{3}$ and $2 n_{3}$. We can split $\mathcal{K}$ into subsets $\mathcal{K}_{1}$ and $\mathcal{K}_{2}$, the first of which contains intervals of depth $n_{3}$ and the second one contains intervals of depth $2 n_{3}$. The indices of the intervals in $\mathcal{K}_{1}$ belong to a set $C_{1} \subset \mathcal{M}_{n_{3}}$. In a similar way, the indices of the intervals in $\mathcal{K}_{2}$ belong to a set $C_{2} \subset \mathcal{M}_{2 n_{3}}$. For $\mathbf{m} \in \mathcal{M}_{2 n_{3}}$, denote by $b(\mathbf{m})$ and $e(\mathbf{m})$ the two words of length $n_{3}$ defined as the beginning and the ending parts of $\mathbf{m}$. Let $C_{3}:=b\left(C_{2}\right)$.

Since $\mathcal{K}$ is a subcover of $\mathcal{J}_{\star}$, the inclusion $\mathcal{M}_{n_{3}} \times \mathcal{M}_{n_{3}} \subset \mathcal{M}_{2 n_{3}}$ proves the relations

$$
C_{3} \cup C_{1}=\mathcal{M}_{n_{3}}, \quad C_{3} \times \mathcal{M}_{n_{3}} \subset C_{2} .
$$

Then we obtain

$$
\begin{aligned}
\Gamma_{\sigma}(\mathcal{K}) & =\sum_{\mathbf{m} \in C_{1}}\left|I_{\mathbf{m}}\right|^{\sigma}+\sum_{\mathbf{m} \in C_{2}}\left|I_{\mathbf{m}}\right|^{\sigma} \geq \sum_{\mathbf{m} \in C_{1}}\left|I_{\mathbf{m}}\right|^{\sigma}+L^{-2 \sigma} \sum_{\mathbf{m} \in C_{2}}\left|I_{b(\mathbf{m})}\right|^{\sigma}\left|I_{e(\mathbf{m})}\right|^{\sigma} \\
& \geq \sum_{\mathbf{m} \in C_{1}}\left|I_{\mathbf{m}}\right|^{\sigma}+L^{-2 \sigma} \sum_{\mathbf{m} \in C_{3}}\left|I_{\mathbf{m}}\right|^{\sigma} \sum_{\mathbf{m} \in \mathcal{M}_{n_{3}}}\left|I_{\mathbf{m}}\right|^{\sigma}
\end{aligned}
$$

and finally, using twice the inequality $\Gamma_{\sigma}\left(\mathcal{J}_{n_{3}}\right)>1$,

$$
\Gamma_{\sigma}(\mathcal{K}) \geq \sum_{\mathbf{m} \in C_{1}}\left|I_{\mathbf{m}}\right|^{\sigma}+L^{-2 \sigma} \Gamma_{\sigma}\left(\mathcal{J}_{n_{3}}\right) \sum_{\mathbf{m} \in C_{3}}\left|I_{\mathbf{m}}\right|^{\sigma} \geq L^{-2 \sigma} \Gamma_{\sigma}\left(\mathcal{J}_{n_{3}}\right) \geq L^{-2 \sigma} .
$$

An inductive argument shows that this result is valid for any cover by intervals whose depth is a multiple of $n_{3}$.

STEP 3. Consider a finite subset $\mathcal{K}$ of $\mathcal{J}_{\star}$ which is a cover of $E$. Its intervals may have variable depth, but its lower depth equals $n_{0}$, with $n_{0}>$ $n_{3}$. Consider the cover $\underline{\mathcal{K}}$ obtained from $\mathcal{K}$ by replacing each fundamental interval $K$ by the fundamental interval $\underline{K}$ containing $K$ and of depth the largest possible multiple of $n_{3}$. This cover is of the form studied in Step 2.

If $d(K)=a n_{3}+r$ with $a \geq 1$ and $0 \leq r<n_{3}$, one has $d(\underline{K})=a n_{3}$, and $|\underline{K}| \leq|K| L^{2} \alpha\left(n_{3}\right)$, and $d(\underline{K}) \geq d(K)-n_{3}+1$. Then, for any $\varepsilon>0$,

$$
\Gamma_{\sigma-\varepsilon}(\underline{\mathcal{K}}) \leq \alpha\left(n_{3}\right)^{\sigma-\varepsilon} L^{2 \sigma-\varepsilon} \Gamma_{\sigma-\varepsilon}(\mathcal{K}) .
$$

Since the lower depth of $\underline{\mathcal{K}}$ is at least $n_{0}-n_{3}+1$, all of the intervals have length smaller than $\delta^{n_{0}-n_{3}}$, and

$$
\Gamma_{\sigma-\varepsilon}(\underline{\mathcal{K}}) \geq \delta^{-\left(n_{0}-n_{3}\right) \varepsilon} \Gamma_{\sigma}(\overline{\mathcal{K}}) .
$$

If we apply the results of Step 2 to $\underline{\mathcal{K}}$, we obtain

$$
\Gamma_{\sigma-\varepsilon}(\underline{\mathcal{K}}) \geq \delta^{-\left(n_{0}-n_{3}\right) \varepsilon} L^{-2 \sigma} \text {. }
$$

Then, by (3.2),

$$
\Gamma_{\sigma-\varepsilon}(\mathcal{K}) \geq \alpha\left(n_{3}\right)^{-(\sigma-\varepsilon)} L^{-\varepsilon} \delta^{-\varepsilon\left(n_{0}-n_{3}\right)},
$$

and choosing $n_{0}$ large enough entails that $\Gamma_{\sigma-\varepsilon}(\mathcal{K})>1$ for any cover $\mathcal{K}$ of 
lower depth larger than $n_{0}$. Then Lemma 6 proves that $\operatorname{dim} E \geq \sigma-\varepsilon$. This is valid for all $\varepsilon>0$, so $\operatorname{dim} E \geq \sigma$.

By Lemmas 4 and 7, Proposition 1 is proven.

3.4. Coming back to the set $F_{M}$. We denote by $A_{n}(M, \sigma)$ and $B_{n}(M, \sigma)$ the associated $\Gamma_{\sigma}$ quantities [defined in (3.1)], related to the constraints $\mathcal{A}_{n}(M), \mathcal{B}_{n}(M)$ defined in (2.7):

$$
A_{n}(M, \sigma):=\sum_{\mathbf{m} \in \mathcal{A}_{n}(M)} p_{\mathbf{m}}^{\sigma}, \quad B_{n}(M, \sigma):=\sum_{\mathbf{m} \in \mathcal{B}_{n}(M)} p_{\mathbf{m}}^{\sigma} .
$$

Note that the cyclic lemma and property (2.1) provide a useful relation between $A_{n}(M, s)$ and $B_{n}(M, s)$ :

$$
B_{n}(M, s) \leq A_{n}(M, s) \leq n \cdot L^{2 s} \cdot B_{n}(M, s) .
$$

This is due to the fact that if $\tau \in \Sigma_{n}$ is a circular permutation, then there exist two inverse branches $h$ and $g$ of $\mathcal{H}^{\star}$ such that $h_{\mathbf{m}}=h \circ g$ and $h_{\tau(\mathbf{m})}=$ $g \circ h$. Now the distortion property entails that $p_{\tau(\mathbf{m})} \leq L^{2} p_{\mathbf{m}}$.

The following result summarizes the results of this section; its proof uses Proposition 1 and Lemma 3.

Corollary 1. Consider a triple $(I, T, c)$ of $\mathcal{G} \mathcal{L G}$-type. For $M>\gamma(c)$, the Hausdorff dimension of the set $F_{M}$ satisfies

$$
\operatorname{dim} F_{M}=\inf \left\{\sigma ; \sup _{n} B_{n}(M, \sigma)<\infty\right\} .
$$

\section{THE MAIN TOOL: THE WEIGHTED TRANSFER OPERATOR}

This section introduces our main tool: the weighted transfer operator. In the following section, this operator will provide useful information on the asymptotic behaviour of the sequences $A_{n}(M, s)$. Here, we summarize its main well-known properties, and, in particular, its dominant spectral properties (Proposition 2). Then we describe more precisely the properties of the operator on the frontier of the domain where it is defined, where analyticity does not hold anymore.

4.1. Transfer operators. Consider a dynamical system $(I, T)$ of the Good Class with a cost $c$. The weighted transfer operator $\mathbf{H}_{\mathbf{m}, s, w}$ relative to a prefix $\mathbf{m} \in \mathcal{M}^{\star}$ is defined as

$$
\mathbf{H}_{\mathbf{m}, s, w}[f](x):=\exp [w c(\mathbf{m})] \cdot\left|h_{\mathbf{m}}^{\prime}(x)\right|^{s} \cdot f \circ h_{\mathbf{m}}(x) \quad(s, w \in \mathbb{C}) .
$$

Due to the additivity of the cost (2.4) and the multiplicativity of the derivative, the operators $\mathbf{H}_{\mathbf{m}, s, w}$ satisfy the fundamental composition property

$$
\mathbf{H}_{\mathbf{m}, s, w} \circ \mathbf{H}_{\mathbf{n}, s, w}=\mathbf{H}_{\mathbf{n} \cdot \mathbf{m}, s, w}
$$

where $\mathbf{n} \cdot \mathbf{m}$ denotes the concatenation of the words $\mathbf{n}$ and $\mathbf{m}$. 
The weighted transfer operator $\mathbf{H}_{s, w}$ is defined as the sum of all $\mathbf{H}_{m, s, w}$ when $m \in \mathcal{M}$, and has already been defined in (1.7):

$$
\mathbf{H}_{s, w}[f](x):=\sum_{m \in \mathcal{M}} \exp [w c(m)] \cdot\left|h_{m}^{\prime}(x)\right|^{s} \cdot f \circ h_{m}(x) .
$$

The composition property (4.1) entails that the $n$th iterate $\mathbf{H}_{s, w}^{n}$ of $\mathbf{H}_{s, w}$ satisfies

$$
\mathbf{H}_{s, w}^{n}=\sum_{\mathbf{m} \in \mathcal{M}^{n}} \mathbf{H}_{\mathbf{m}, s, w} \quad \text { for any } n \geq 1 .
$$

For $w=0$, the operator $\mathbf{H}_{\mathbf{m}, s, w}$ coincides with the classical transfer operator

$$
\mathbf{H}_{\mathbf{m}, s}[f](x):=\left|h_{\mathbf{m}}^{\prime}(x)\right|^{s} \cdot f \circ h_{\mathbf{m}}(x),
$$

which is closely related to the Lebesgue measure $p_{\mathbf{m}}$ of the fundamental interval $I_{\mathbf{m}}$ : the length $p_{\mathbf{m}}$ satisfies $p_{\mathbf{m}}^{s}=\left|h_{\mathbf{m}}(0)-h_{\mathbf{m}}(1)\right|^{s}=\left|h_{\mathbf{m}}^{\prime}\left(\theta_{\mathbf{m}}\right)\right|^{s}$ for some $\left.\theta_{\mathbf{m}} \in\right] 0,1[$, and the distortion property (2.1) entails that the sequence

$$
D_{n}(M, s):=\sum_{\mathbf{m} \in \mathcal{A}_{n}(M)} \mathbf{H}_{\mathbf{m}, s}[1](0)
$$

is related to the sequence $A_{n}(M, s)$ [defined in (3.3)] via

$$
L^{-s} A_{n}(M, s) \leq D_{n}(M, s) \leq L^{s} A_{n}(M, s) .
$$

Finally, by (3.4) and (4.3), the three sequences $A_{n}(M, s), B_{n}(M, s)$ and $D_{n}(M, s)$ are closely related:

$$
\begin{aligned}
L^{-s} B_{n}(M, s) & \leq L^{-s} A_{n}(M, s) \leq D_{n}(M, s) \\
& \leq L^{s} A_{n}(M, s) \leq L^{3 s} n B_{n}(M, s) .
\end{aligned}
$$

4.2. Functional analysis and spectral properties. We next recall some well-known spectral properties of the transfer operator $\mathbf{H}_{s, w}$ [see [2] for a complete treatment of transfer operators]. Endow the Banach space $\mathcal{C}^{1}(I)$ with the norm $\|\cdot\|_{1}$ defined by

$$
\|f\|_{1}:=\sup \{|f(t)| ; t \in I\}+\sup \left\{\left|f^{\prime}(t)\right| ; t \in I\right\} .
$$

When $(\sigma:=\Re s, \varrho:=\Re w)$ belongs to the set

$$
\mathcal{S}:=\left\{(\sigma, \varrho) ; \sum_{m \in \mathcal{M}} \exp [\varrho c(m)] \delta_{m}^{\sigma}<\infty\right\}
$$

the norm $\left\|\mathbf{H}_{s, w}\right\|_{1}$ is bounded and $\mathbf{H}_{s, w}$ acts on $\mathcal{C}^{1}(I)$. For a triple $(I, T, c)$ of $\mathcal{G} \mathcal{L G}$, one has

$$
\mathcal{S} \supseteq \mathcal{S}_{1} \quad \text { with } \quad \mathcal{S}_{1}:=\{(\sigma, \varrho) ; \sigma \geq 0, \varrho<0\} \cup\left\{(\sigma, 0) ; \sigma>\sigma_{0}\right\},
$$

and condition (c3) of Section 2.4 proves that $\mathcal{S}$ cannot contain any $(\sigma, \varrho)$ with $\varrho>0$.

Generally speaking, $\mathbf{H}_{s, w}$ is not compact acting on $\mathcal{C}^{1}(I)$; however, it is quasi-compact. We recall the definition of quasi-compactness for a bounded 
operator $\mathbf{L}$ on a Banach space. Denote by $\operatorname{Sp}(\mathbf{L})$ the spectrum of $\mathbf{L}$, by $R(\mathbf{L})$ its spectral radius, and by $R_{\mathrm{e}}(\mathbf{L})$ its essential spectral radius, i.e., the smallest $r \geq 0$ such that any $\lambda \in \operatorname{Sp}(\mathbf{L})$ with $|\lambda|>r$ is an isolated eigenvalue of finite multiplicity. An operator $\mathbf{L}$ is quasi-compact if $R_{\mathrm{e}}(\mathbf{L})<R(\mathbf{L})$.

We denote the partial derivatives of first and second order of a function $F(s, w)$ at $(a, b)$ by $F_{w}^{\prime}(a, b), F_{s}^{\prime}(a, b), F_{w^{2}}^{\prime \prime}(a, b), F_{s^{2}}^{\prime \prime}(a, b), F_{w s}^{\prime \prime}(a, b)$.

Proposition 2 [Classical spectral properties of transfer operators]. Let $\mathbf{H}_{s, w}$ be the transfer operator (1.7) associated to a $\mathcal{G} \mathcal{L} \mathcal{G}$ triple $(I, T, c)$ with contraction constant $\delta$. Denote by $R(s, w)$ its spectral radius and by $R_{\mathrm{e}}(s, w)$ its essential spectral radius. Denote by $\operatorname{Int}\left(\mathcal{S}_{1}\right)$ the interior of the domain $\mathcal{S}_{1}$ defined in (4.5).

(1) [Quasi-compactness] If $(\sigma=\Re s, \varrho=\Re w) \in \mathcal{S}_{1}$, then $\mathbf{H}_{s, w}$ acts boundedly on $\mathcal{C}^{1}(I)$. In that case $R(s, w) \leq R(\sigma, \varrho)$ and $R_{\mathrm{e}}(s, w) \leq$ $\delta^{\sigma} \cdot R(\sigma, \varrho)$; in particular $\mathbf{H}_{s, w}$ is quasi-compact for real $(s, w)$.

(2) [Unique dominant eigenvalue] For real $(s, w) \in \mathcal{S}_{1}, \mathbf{H}_{s, w}$ has a unique eigenvalue $\lambda(s, w)$ of maximal modulus, which is real and simple, the dominant eigenvalue. The associated eigenfunction $f_{s, w}$ is strictly positive, the associated eigenvector $\nu_{s, w}$ of the adjoint operator $\mathbf{H}_{s, w}^{*}$ is a positive Radon measure, and, with the normalization conditions $\nu_{s, w}[1]=1$ and $\nu_{s, w}\left[f_{s, w}\right]=1$, the pair $\left(f_{s, w}, \nu_{s, w}\right)$ is unique.

(3) [Spectral gap] For real $(s, w) \in \mathcal{S}_{1}$, there is a spectral gap, i.e., the subdominant spectral radius $r_{s, w} \geq R_{\mathrm{e}}(s, w)$ defined by

$$
r_{s, w}:=\sup \left\{|\lambda| ; \lambda \in \operatorname{Sp}\left(\mathbf{H}_{s, w}\right), \lambda \neq \lambda(s, w)\right\},
$$

satisfies $r_{s, w}<\lambda(s, w)$.

(4) [Analyticity with respect to $(s, w)]$ The operator $\mathbf{H}_{s, w}$ depends analytically on $(s, w)$ for $(\Re s, \Re w) \in \operatorname{Int}\left(\mathcal{S}_{1}\right)$. For any real $(\sigma, \varrho) \in$ $\operatorname{Int}\left(\mathcal{S}_{1}\right)$, there exists a (complex) neighbourhood $\mathcal{V}$ of $(\sigma, \varrho)$ on which $\lambda(s, w)^{ \pm 1}, f_{s, w}^{ \pm 1}$, and $f_{s, w}^{\prime}$ depend analytically on $(s, w)$.

(5) [Analyticity with respect to $s$ when $w=0$ ] When $w=0$, we omit the second index in the operator and its associated objects. For real $s>\sigma_{0}$, the operator $\mathbf{H}_{s}$ depends analytically on $s$, and $s \mapsto \lambda(s)^{ \pm 1}$ and $s \mapsto f_{s}^{ \pm 1}$ are analytic. For $(s, w)=(1,0)$, the operator $\mathbf{H}_{s, w}$ coincides with the density transformer. Thus, the dominant spectral objects of $\mathbf{H}_{1,0}$ satisfy:

$$
\begin{aligned}
\lambda(1,0)=1, \quad f_{1,0} & =f_{1}=\text { stationary density } \\
\nu_{1,0} & =\nu_{1}=\text { Lebesgue measure. }
\end{aligned}
$$

(6) [Derivatives of the pressure] For real $(s, w) \in \mathcal{S}_{1}$, define the pressure function $\Lambda(s, w)=\log \lambda(s, w)$. For real $(s, w) \in \operatorname{Int}\left(\mathcal{S}_{1}\right)$, its deriva- 
tives satisfy

$$
\Lambda_{s}^{\prime}(s, w) \leq \log \eta_{1}<0, \quad \Lambda_{w}^{\prime}(s, w) \geq \gamma(c)>0 .
$$

Furthermore, the quantity $\Lambda_{s}^{\prime}(1,0)$ equals the opposite of the entropy $h$ of the system. The map $(s, w) \mapsto \Lambda(s, w)$ is strictly convex.

REMARK. The strict convexity of the map does not entail that the second derivative $\Lambda_{w^{2}}^{\prime \prime}(s, w)$ is always non-zero for real $(s, w) \in \mathcal{S}_{1}$. We shall prove this last assertion in Section 5 (Lemma 14).

4.3. Quasi-powers property. For complex $(s, w)$ sufficiently near $\operatorname{Int}\left(\mathcal{S}_{1}\right)$, the operator $\mathbf{H}_{s, w}$ decomposes as

$$
\mathbf{H}_{s, w}=\lambda(s, w) \mathbf{P}_{s, w}+\mathbf{N}_{s, w} .
$$

Here, $\mathbf{P}_{s, w}$ defined by $\mathbf{P}_{s, w}[f](x):=f_{s, w}(x) \nu_{s, w}[f]$ is the projection on the dominant eigenspace, the spectral radius $r_{s, w}$ of $\mathbf{N}_{s, w}$ is strictly less than $|\lambda(s, w)|$, and $\mathbf{P}_{s, w} \circ \mathbf{N}_{s, w}=\mathbf{N}_{s, w} \circ \mathbf{P}_{s, w}=0$. For any $\alpha>r_{s, w} /|\lambda(s, w)|$, and for any $f \in \mathcal{C}^{1}(I), f>0$, we have the quasi-powers property

$$
\mathbf{H}_{s, w}^{n}[f](x)=\lambda(s, w)^{n} \mathbf{P}_{s, w}[f](x)\left[1+O\left(\alpha^{n}\right)\right]
$$

for $x \in I$ and $(s, w)$ near $\operatorname{Int}\left(\mathcal{S}_{1}\right)$.

It is important to describe the behaviour of spectral objects on the frontier of $\mathcal{S}_{1}$, when $s>\sigma_{0}$ and $w \rightarrow 0^{-}$or $w \rightarrow-\infty$. Since the function $w \mapsto \Lambda_{w}^{\prime}(s, w)$ is strictly increasing, it has both limits as $w \rightarrow 0^{-}$and as $w \rightarrow-\infty$. We let

$$
\beta_{0}(s):=\lim _{w \rightarrow 0^{-}} \Lambda_{w}^{\prime}(s, w), \quad \beta_{\infty}(s):=\lim _{w \rightarrow-\infty} \Lambda_{w}^{\prime}(s, w) .
$$

The next three subsections will be devoted to describing the behaviour of spectral objects on the frontier of $\mathcal{S}_{1}$.

4.4. Behaviour of $\Lambda_{w}^{\prime}(s, w)$ for $w \rightarrow-\infty$. We first study $\beta_{\infty}(s)$.

Lemma 8. For any triple of $\mathcal{G} \mathcal{L G}$-type, and for any $s \geq 0$, the derivative $\Lambda_{w}^{\prime}(s, w)$ tends to $\gamma(c)$ as $w \rightarrow-\infty$. Equivalently, $\beta_{\infty}(s)=\gamma(c)$.

Proof. First, since $\left\{x ; C_{n}(x)<\gamma(c) n\right\}=\emptyset$, we have

$$
\mathbf{H}_{s, w}^{n}[1](0)=\sum_{r \geq \gamma n} e^{r w} \sum_{\mathbf{m} \in \mathcal{M}^{n}, c(\mathbf{m})=r} \mathbf{H}_{\mathbf{m}, s}[1](0) .
$$

Consider now some $w_{0}<0$. Then, for any $w<w_{0}$,

$$
\sum_{r \geq \gamma n} e^{r w} \sum_{\mathbf{m} \in \mathcal{M}^{n}, c(\mathbf{m})=r} \mathbf{H}_{\mathbf{m}, s}[1](0) \leq e^{\gamma n\left(w-w_{0}\right)} \mathbf{H}_{s, w_{0}}^{n}[1](0) .
$$


Finally, by the quasi-powers property, there exist two constants $d_{1}$ and $d_{2}$ that depend on $s, w$ but not on $n$ so that, for $n$ large enough,

$$
d_{1} \lambda(s, w)^{n} \leq \mathbf{H}_{s, w}^{n}[1](0) \leq e^{\gamma n\left(w-w_{0}\right)} \mathbf{H}_{s, w_{0}}^{n}[1](0) \leq d_{2} e^{\gamma n\left(w-w_{0}\right)} \lambda\left(s, w_{0}\right)^{n} .
$$

Then, for any $(s, w)$ fixed in $\mathcal{S}_{1}$ with $w<w_{0}$, the sequence

$$
\left(\frac{\lambda(s, w)}{e^{\gamma\left(w-w_{0}\right)} \lambda\left(s, w_{0}\right)}\right)^{n}
$$

is bounded. This proves that $\Lambda(s, w) \leq \gamma\left(w-w_{0}\right)+\Lambda\left(s, w_{0}\right)$, and finally

$$
\liminf _{w \rightarrow-\infty} \frac{\Lambda(s, w)}{w} \geq \gamma .
$$

On the other hand, recall that the minimum value $\gamma(c)$ of $c(m)$ is attained at $m=1$. The length $p_{\mathbf{m}}$ of the fundamental interval relative to the sequence $\mathbf{m}=(1, \ldots, 1)$ ( $n$ times) satisfies $p_{\mathbf{m}} \geq \eta_{1}^{n}$. Thus, the inequality $\mathbf{H}_{s, w}^{n}[1](0) \geq e^{n \gamma w} p_{\mathbf{m}}^{s}$ together with the quasi-power property entails the existence of a positive constant $d_{4}$ such that $d_{4} \lambda(s, w)^{n} \geq e^{n \gamma w} \eta_{1}^{s n}$. Hence, for any $(s, w)$ fixed in $\mathcal{S}_{1}$, the sequence

$$
\left(\frac{e^{\gamma w} \eta_{1}^{s}}{\lambda(s, w)}\right)^{n}
$$

is bounded. This proves that $\Lambda(s, w) \geq \gamma w+s \log \eta_{1}$ and finally

$$
\limsup _{w \rightarrow-\infty} \frac{\Lambda(s, w)}{w} \leq \gamma .
$$

Finally, by (4.8) and (4.9) and L'Hôpital's rule, the lemma is proven.

In the $\mathcal{G} \mathcal{L G}$-setting, the behaviour of the operator $\mathbf{H}_{s, w}$ when $w \rightarrow 0^{-}$is not a priori clear, and has to be made more precise. We are mainly interested in studying the behaviour of the dominant spectral objects when $s$ is fixed $\left(s>\sigma_{0}\right)$ and $w \rightarrow 0^{-}$.

\subsection{Explicit expression of the dominant eigenvalue and its} derivatives. In the case when the branches are affine, the eigenfunction $f_{s, w}$ equals 1 , and the eigenvalue $\lambda(s, w)$ is explicit,

$$
\lambda(s, w)=\sum_{m \in \mathcal{M}} \exp [w c(m)] p_{m}^{s} .
$$

It is thus easy to obtain explicit expressions for the derivatives

$$
\begin{aligned}
& \lambda_{w}^{\prime}(s, w)=\sum_{m \in \mathcal{M}} c(m) \exp [w c(m)] p_{m}^{s}, \\
& \lambda_{s}^{\prime}(s, w)=\sum_{m \in \mathcal{M}} \exp [w c(m)] \log p_{m} \cdot p_{m}^{s} .
\end{aligned}
$$

In the general case, the expressions for $\lambda$ and its derivatives involve the 
sequence of integrals

$$
\begin{aligned}
& I_{m}(s, w):=\int_{I}\left|h_{m}^{\prime}(t)\right|^{s} \cdot f_{s, w} \circ h_{m}(t) d \nu_{s, w}(t), \\
& J_{m}(s, w):=\int_{I} \log \left|h_{m}^{\prime}(t)\right| \cdot\left|h_{m}^{\prime}(t)\right|^{s} \cdot f_{s, w} \circ h_{m}(t) d \nu_{s, w}(t),
\end{aligned}
$$

and the following holds.

Lemma 9. The dominant eigenvalue and its first derivatives admit the following expressions:

$$
\begin{array}{rlrl}
\lambda(s, w) & =\sum_{m \in \mathcal{M}} \exp [w c(m)] I_{m}(s, w) & \text { for }(s, w) \in \mathcal{S}_{1}, \\
\lambda_{w}^{\prime}(s, w) & =\sum_{m \in \mathcal{M}} c(m) \exp [w c(m)] I_{m}(s, w) & & \text { for }(s, w) \in \operatorname{Int}\left(\mathcal{S}_{1}\right), \\
\lambda_{s}^{\prime}(s, w) & =\sum_{m \in \mathcal{M}} \exp [w c(m)] J_{m}(s, w) & & \text { for }(s, w) \in \mathcal{S}_{1},
\end{array}
$$

which involve the integrals $I_{m}(s, w), J_{m}(s, w)$ defined in (4.12) and (4.13).

Proof. Consider a point $(s, w) \in \mathcal{S}_{1}$. Integrating the relation $\mathbf{H}_{s, w}\left[f_{s, w}\right]$ $=\lambda(s, w) f_{s, w}$ with respect to the measure $\nu_{s, w}$ provides the first result. Consider now a point $(s, w)$ in the interior of $\mathcal{S}_{1}$. All the quantities that appear in the relation $\mathbf{H}_{s, w}\left[f_{s, w}\right]=\lambda(s, w) f_{s, w}$ are analytic with respect to $w$ at $(s, w)$. Differentiating this relation with respect to $w$ gives

$$
\frac{d}{d w} \mathbf{H}_{s, w}\left[f_{s, w}\right]+\mathbf{H}_{s, w}\left[\frac{d}{d w} f_{s, w}\right]=\lambda_{w}^{\prime}(s, w) f_{s, w}+\lambda(s, w) \frac{d}{d w} f_{s, w} .
$$

The definition of $\nu_{s, w}$ provides the equality

$$
\int_{I} \mathbf{H}_{s, w}[g](t) d \nu_{s, w}(t)=\lambda(s, w) \int_{I} g(t) d \nu_{s, w}(t) \quad \text { for any } g \in \mathcal{C}^{1}(I) .
$$

Apply it to $g:=\frac{d}{d w} f_{s, w}$. Then, by (4.17) and (4.18) and the normalization condition,

$$
\int_{I} \frac{d}{d w} \mathbf{H}_{s, w}\left[f_{s, w}\right](t) d \nu_{s, w}(t)=\lambda_{w}^{\prime}(s, w) \int_{I} f_{s, w}(t) d \nu_{s, w}(t)=\lambda_{w}^{\prime}(s, w) .
$$

In the same vein, for $(s, w) \in \mathcal{S}_{1}$,

$$
\int_{I} \frac{d}{d s} \mathbf{H}_{s, w}\left[f_{s, w}\right](t) d \nu_{s, w}(t)=\lambda_{s}^{\prime}(s, w) \int_{I} f_{s, w}(t) d \nu_{s, w}(t)=\lambda_{s}^{\prime}(s, w) .
$$

4.6. Behaviour of $\Lambda_{w}^{\prime}(s, w)$ when $w \rightarrow 0^{-}$. Since $w \mapsto \Lambda_{w}^{\prime}(s, 0)$ is increasing, its limit (possibly infinite) exists as $w \rightarrow 0^{-}$. On the other hand, the quantity $\Lambda_{w}^{\prime}(s, 0)$ is well-defined (possibly infinite for $s \leq \sigma_{1}$ ). The main question is: Are they equal? In other words, is $w \mapsto \Lambda_{w}^{\prime}(s, w)$ continuous at $w=0$ ? The following lemma provides a positive answer to this question. 
LEMMA 10. For any triple of $\mathcal{G} \mathcal{L} \mathcal{G}$-type, the following holds:

(a) For $s>\sigma_{0}$, the map $(s, w) \mapsto \mathbf{H}_{s, w}$ is continuous at $\left(s, 0^{-}\right)$. So, all the dominant spectral objects are continuous at $\left(s, 0^{-}\right)$. Furthermore, the map $(s, w) \mapsto \Lambda_{s}^{\prime}(s, w)$ is continuous at $\left(s, 0^{-}\right)$.

(b) For $s>\sigma_{1}$, the function $w \mapsto \Lambda_{w}^{\prime}(s, w)$ is continuous at $w=0^{-}$, and

$$
\beta_{0}(s)=\frac{1}{\lambda(s)} \sum_{m \in \mathcal{M}} c(m) I_{m}(s, 0), \quad \beta_{0}(1)=\sum_{m \in \mathcal{M}} c(m) \bar{p}_{m}=\mu(c) .
$$

(c) For $\sigma_{0}<s \leq \sigma_{1}$, the function $w \mapsto \Lambda_{w}^{\prime}(s, w)$ tends to $\infty$ as $w \rightarrow 0^{-}$.

Proof. We deal with the memoryless dynamical system which provides an approximation of the behaviour of our dynamical system, and all the quantities relative to this memoryless approximation will be marked with a hat. We shall relate the dominant eigenvalue $\lambda(s, w)$, together with its derivatives, to their analogues in the approximate model. Recall that relations (4.10) and (4.11) provide explicit expressions for $\widehat{\lambda}$ and its derivatives which involve the length $p_{m}$ of fundamental intervals. These relations show that any of the four objects

$$
\widehat{\lambda}(s, w), \quad \widehat{\lambda}_{w}^{\prime}(s, w), \quad-\widehat{\lambda}_{s}^{\prime}(s, w), \quad \widehat{\lambda}_{w^{2}}^{\prime \prime}(s, w)
$$

is the sum of a series whose general term is a positive increasing function of $w$. Thus,

$$
\lim _{w \rightarrow 0^{-}} \widehat{\lambda}(s, w)=\widehat{\lambda}(s, 0), \quad \lim _{w \rightarrow 0^{-}} \widehat{\lambda}_{s}^{\prime}(s, w)=\widehat{\lambda}_{s}^{\prime}(s, 0),
$$

these two terms being infinite when $s \leq \sigma_{0}$, and

$$
\lim _{w \rightarrow 0^{-}} \widehat{\lambda}_{w}^{\prime}(s, w)=\widehat{\lambda}_{w}^{\prime}(s, 0)
$$

this last term being infinite when $s \leq \sigma_{1}$.

(a) For $s>\sigma_{0}$, the map $(s, w) \mapsto \mathbf{H}_{s, w}$ is continuous at $(s, 0)$ : In the decomposition

$$
\left\|\mathbf{H}_{t, w}-\mathbf{H}_{s, 0}\right\|_{1} \leq\left\|\mathbf{H}_{t, w}-\mathbf{H}_{t, 0}\right\|_{1}+\left\|\mathbf{H}_{t, 0}-\mathbf{H}_{s, 0}\right\|_{1} \quad \text { for }(t, w) \text { near }(s, 0),
$$

the second term on the right hand side equals $\left\|\mathbf{H}_{t}-\mathbf{H}_{s}\right\|_{1}$ while the first term can be compared, via the distortion property, to the corresponding term for the dominant eigenvalue $\widehat{\lambda}$ :

$$
\left\|\mathbf{H}_{t, w}-\mathbf{H}_{t, 0}\right\|_{1} \leq K|\widehat{\lambda}(t, w)-\widehat{\lambda}(t, 0)|
$$

for some constant $K$. This proves, by (4.19), for any $s>\sigma_{0}$, the continuity of $(s, w) \mapsto \mathbf{H}_{s, w}$ at $(s, 0)$. Then, perturbation theory [30] (with continuous perturbation, and not analytic) is applied to the quasi-compact operator $\mathbf{H}_{s, w}$ near $(s, 0)$, and proves that the dominant spectral objects are continuous at $(s, 0)$. In particular, the maps $(s, w) \mapsto \lambda(s, w),(s, w) \mapsto f_{s, w}$, 
$(s, w) \mapsto \nu_{s, w}$ are continuous at $(s, 0)$. For $s>\sigma_{0}$, an upper bound for the difference $\left|\lambda_{s}^{\prime}(t, w)-\lambda_{s}^{\prime}(s, 0)\right|$ is

$$
\sum_{m \geq 1}(1-\exp [w c(m)]) I_{m}(t, 0)+\left|\lambda_{s}^{\prime}(t, 0)-\lambda_{s}^{\prime}(s, 0)\right| .
$$

Since $\lambda_{s^{2}}^{\prime \prime}$ is bounded, the second term is $O(|s-t|)$. Since the map $f_{t}$ admits an upper bound $b>0$, one has $I_{m}(t, 0) \leq L^{t} b p_{m}^{t}$, and an upper bound for the first term is $L^{t} b\left|\widehat{\lambda}_{w}^{\prime}(t, w)-\widehat{\lambda}_{w}^{\prime}(t, 0)\right|$; this proves, by (4.20), the continuity of $(s, w) \mapsto \lambda_{s}^{\prime}(s, w)$ at $(s, 0)$. Since $(s, w) \mapsto \lambda(s, w)$ is also continuous at $(s, 0)$, the last assertion of (a) is proven.

(b) For $s>\sigma_{1}$, the quantity

$$
\left\|\frac{\partial}{\partial w} \mathbf{H}_{s, w}-\frac{\partial}{\partial w} \mathbf{H}_{s, 0}\right\|_{1}
$$

is, up to a positive multiplicative constant independent of $w$, upper-bounded by the difference $\left|\widehat{\lambda}_{w}^{\prime}(s, w)-\widehat{\lambda}_{w}^{\prime}(s, 0)\right|$ which tends to zero as $w \rightarrow 0^{-}$. Thus, the map $w \mapsto \mathbf{H}_{s, w}$ is of class $\mathcal{C}^{1}$ at $w=0^{-}$. By perturbation theory, the same holds for its dominant spectral objects, and the function $w \mapsto \Lambda_{w}^{\prime}(s, w)$ is continuous at $w=0^{-}$.

(c) For $\sigma_{0}<s \leq \sigma_{1}$, consider a compact neighbourhood $\mathcal{S}_{2}$ of a point $(s, 0)$ in $\mathcal{S}_{1}$. The mapping $(s, w, t) \mapsto f_{s, w}(t)$ is continuous on the compact set $\mathcal{S}_{2} \times I$ and strictly positive. It admits a lower bound $a>0$, and the distortion property entails that

$$
\lambda_{w}^{\prime}(s, w) \geq \frac{a}{L^{s}} \widehat{\lambda}_{w}^{\prime}(s, w),
$$

and $\lambda_{w}^{\prime}(s, w)$ tends to $\infty$ as $w \rightarrow 0^{-}$.

\section{HAUSDORFF DIMENSION AND DOMINANT EIGENVALUES}

This section is devoted to proving Theorem 1 which relates the Hausdorff dimension to the solution of a differential system that involves the dominant eigenvalue of the weighted transfer operator. The proof makes use of tools that are often applied for proving large deviation results, since it strongly uses the quasi-powers theorem together with a well-known technique called "shifting of the mean" [7].

We use the sequence $D_{n}(M, s)$ defined in (4.2), and we wish to relate it to the dominant eigenvalue $\lambda(s, w)$ of the weighted operator $\mathbf{H}_{s, w}$. More precisely, for any $s \in[0,1]$ and $w<0$ we introduce the quantities

$$
A_{M, s}(w):=\exp [-M w] \cdot \lambda(s, w), \quad \alpha_{M}(s):=\inf \left\{A_{M, s}(w) ; w<0\right\}
$$

and we will prove in Section 5.2 that the sequence $\left[D_{n}(M, s)\right]^{1 / n}$ tends to $\alpha_{M}(s)$. 
5.1. Minimum of $w \mapsto \exp [-M w] \cdot \lambda(s, w)$. We first study the function $\log A_{M, s}$. Its derivative is the strictly increasing function $\Lambda_{w}^{\prime}(s, w)-M$ which varies from $\beta_{\infty}(s)-M$ to $\beta_{0}(s)-M$. The function $A_{M, s}$ admits a minimum in $]-\infty, 0[$ if and only if

$$
\beta_{\infty}(s)<M<\beta_{0}(s) .
$$

Suppose that $M \in] \gamma(c), \mu(c)[$. Then the left inequality above always holds by Lemma 8 . On the other hand, Lemma 10 proves that $\beta_{0}(1)=\mu(c)$. Note that, for $s>\sigma_{0}, s \mapsto \beta_{0}(s)$ is continuous, and, for $M<\mu(c)$, denote by $\mathcal{V}_{M}$ the intersection of $[0,1]$ with the largest neighbourhood of $s=1$ for which $\beta_{0}(s)>M$. For $s \leq \sigma_{0}$, one has $\beta_{0}(s)=+\infty$, and there are two cases.

CASE (i). If $\beta_{0}(s)>M$ for any $\left.\left.s \in\right] \sigma_{0}, 1\right]$, then $\mathcal{V}_{M}=[0,1]$; this is the case when $\mu(c)=+\infty$.

CASE (ii). If there exists $\left.s \in] \sigma_{0}, 1\right]$ for which $\beta_{0}(s) \leq M$, we denote by $t_{M}$ the largest $s$ for which $\beta_{0}(s)=M$, and then $\left.\left.\mathcal{V}_{M}=\right] t_{M}, 1\right]$.

Finally, we have proven the following:

Lemma 11. Consider a triple of $\mathcal{G} \mathcal{L G}$-type and $M \in] \gamma(c), \mu(c)[$. Denote by $\mathcal{V}_{M}$ the intersection of $[0,1]$ with the largest neighbourhood of $s=1$ for which $\lim _{w \rightarrow 0^{-}} \Lambda_{w}^{\prime}(s, w)>M$. For any $s \in \mathcal{V}_{M}$, the function $A_{M, s}$ : ]$-\infty, 0[\rightarrow \mathbb{R}$ that associates to $w$ the quantity $\exp [-M w] \lambda(s, w)$ attains its minimum [denoted by $\left.\alpha_{M}(s)\right]$ at $w=\eta(M, s)$, where $\eta(M, s)$ is the unique strictly negative value of $w$ for which $\Lambda_{w}^{\prime}(s, w)=M$.

5.2. Relation between $D_{n}(M, s)$ and $\alpha_{M}(s)$. The following result is central to the proof of Theorem 1 . It relates the sequence $D_{n}(M, s)$ defined in Section 4.1 and $\alpha_{M}(s)$ defined in Lemma 11.

Proposition 3. Consider a triple of $\mathcal{G} \mathcal{L G}$-type, and a pair $(s, M)$ which satisfies $\gamma(c)<M<\mu(c)$ and $s \in \mathcal{V}_{M}$. Then the sequence $\left[D_{n}(M, s)\right]^{1 / n}$ has a limit as $n \rightarrow \infty$ and this limit equals $\alpha_{M}(s)$. Both sequences $\left[A_{n}(M, s)\right]^{1 / n}$ and $\left[B_{n}(M, s)\right]^{1 / n}$ converge to $\alpha_{M}(s)$.

REMARK. The sequences $A_{n}(M, s)$ and $B_{n}(M, s)$ are defined in Section 2.6, and (4.4) proves that both $\left[A_{n}(M, s)\right]^{1 / n}$ and $\left[B_{n}(M, s)\right]^{1 / n}$ have the same limit as $\left[D_{n}(M, s)\right]^{1 / n}$.

Proof. There are three main steps in the proof. The main idea is to relate the sequence $D_{n}(M, s)$ to the $n$th iterate of the weighted transfer operator $\exp [-w M] \mathbf{H}_{s, w}$, and more particularly to its dominant eigenvalue $\exp [-w M n] \lambda^{n}(s, w)$. Lemma 12 provides a first relation between $D_{n}(M, s)$ and $\exp [-w M n] \lambda^{n}(s, w)$ which involves some probability $\Pi_{n}$ related to a $\operatorname{cost} C_{n}$. Lemma 13 proves that the $\operatorname{cost} C_{n}$ asymptotically follows a Gaussian law, which entails an evaluation of $\Pi_{n}$. However, the previous assertion 
is only true if the second derivative $\Lambda_{w^{2}}^{\prime \prime}(s, w)$ is not zero, which is proven in Lemma 14. A priori, in Lemma 12, it is sufficient to deal with a constant density $g=1$. But proving Lemma 14 needs similar arguments to the proof of Lemma 12, where the dominant eigenfunction $f_{s, w}$ of $\mathbf{H}_{s, w}$ replaces the constant density 1 . This is why the following lemma deals with a general density $g$.

Lemma 12. Let $d \nu=g d \nu_{s, w}$ be a probability measure where $g$ is a density with respect to $\nu_{s, w}$ of class $\mathcal{C}^{1}$, and denote by $\|\cdot\|_{\infty}$ the supremum norm. Consider any $(s, w) \in \operatorname{Int}\left(\mathcal{S}_{1}\right)$. Then

$$
\frac{L^{-s}}{\|g\|_{\infty}} e^{w \sqrt{n}} \Pi_{n}\left(\frac{\lambda(s, w)}{e^{M w}}\right)^{n} \leq D_{n}(M, s) \leq a(s, w)\left(\frac{\lambda(s, w)}{e^{M w}}\right)^{n} .
$$

Here $a(s, w)$ is some positive function of $s, w$, and $\Pi_{n}$ is the probability of the event $\left[M n-\sqrt{n} \leq C_{n} \leq M n\right]$ with respect to some measure $\nu$ absolutely continuous with respect to the dominant eigenmeasure $\nu_{s, w}$ of the operator $\mathbf{H}_{s, w}^{\star}$.

Proof. First, note that, for any $w \leq 0$,

$$
\exp [-w M n] \mathbf{H}_{s, w}^{n}[1](0)=\sum_{\mathbf{m} \in \mathcal{M}^{n}} \exp [w(c(\mathbf{m})-M|\mathbf{m}|)] \mathbf{H}_{\mathbf{m}, s}[1](0) .
$$

If $\mathbf{m} \in \mathcal{A}_{n}(M)$ and $w \leq 0$, then $w(c(\mathbf{m})-M|\mathbf{m}|) \geq 0$ and $\exp [w(c(\mathbf{m})-$ $M|\mathbf{m}|)] \geq 1$, so that

$$
D_{n}(M, s):=\sum_{\mathbf{m} \in \mathcal{A}_{n}(M)} \mathbf{H}_{\mathbf{m}, s}[1](0) \leq e^{-w M n} \mathbf{H}_{s, w}^{n}[1](0) .
$$

For any $(s, w) \in \operatorname{Int}\left(\mathcal{S}_{1}\right)$, the quasi-powers property (4.7) ensures that there exists a bounded function $a(s, w)$ such that

$$
D_{n}(M, s) \leq a(s, w)\left(\frac{\lambda(s, w)}{e^{M w}}\right)^{n},
$$

and the upper bound of the lemma is proven.

We now establish the lower bound. For $\mathbf{m} \in \mathcal{M}^{n}$, we consider the probability $\nu\left(I_{\mathbf{m}}\right)$ of the fundamental interval $I_{\mathbf{m}}$,

$$
\int_{I} \mathbf{1}_{h_{\mathbf{m}}(I)} d \nu=\nu_{s, w}\left[g \mathbf{1}_{h_{\mathbf{m}}(I)}\right]=\lambda(s, w)^{-n} \nu_{s, w}\left[\mathbf{H}_{s, w}^{n}\left[g \mathbf{1}_{h_{\mathbf{m}}(I)}\right]\right] .
$$

This entails the equality

$$
\lambda(s, w)^{n} \nu\left(I_{\mathbf{m}}\right)=\int_{I} \mathbf{H}_{\mathbf{m}, s, w}[g] d \nu_{s, w},
$$

which involves the component $\mathbf{H}_{\mathbf{m}, s, w}$ of the operator $\mathbf{H}_{s, w}^{n}$ relative to the 
prefix m. Together with the relation

$$
\begin{aligned}
\int_{I} \mathbf{H}_{\mathbf{m}, s, w}[g] d \nu_{s, w} & =\exp [w c(\mathbf{m})] \int_{I} \mathbf{H}_{\mathbf{m}, s}[g] d \nu_{s, w} \\
& \leq\|g\|_{\infty} \exp [w c(\mathbf{m})] L^{s} \mathbf{H}_{\mathbf{m}, s}[1](0)
\end{aligned}
$$

this relates $\nu\left(I_{\mathbf{m}}\right)$ and the term $\mathbf{H}_{\mathbf{m}, s}[1](0)$ of $D_{n}(M, s)$ :

$$
\mathbf{H}_{\mathbf{m}, s}[1](0) \geq \frac{L^{-s}}{\|g\|_{\infty}} \exp [-w c(\mathbf{m})] \lambda(s, w)^{n} \nu\left(I_{\mathbf{m}}\right)
$$

We now relate $D_{n}(M, s)$ and $\Pi_{n}:=\mathbb{P}_{\nu}\left[M n-\sqrt{n} \leq C_{n} \leq M n\right]$. Since the event $\left[C_{n} \leq M n\right]$ contains $\left[M n-\sqrt{n} \leq C_{n} \leq M n\right]$, one finally obtains

$$
D_{n}(M, s) \geq \frac{L^{-s}}{\|g\|_{\infty}} \exp [-w(M n-\sqrt{n})] \Pi_{n} \lambda(s, w)^{n} .
$$

LEMMA 13. Consider any $(s, w) \in \operatorname{Int}\left(\mathcal{S}_{1}\right)$, and any probability $\nu$ absolutely continuous with respect to the dominant eigenmeasure $\nu_{s, w}$ of the operator $\mathbf{H}_{s, w}^{\star}$. Then the mean and variance of the cost $C_{n}$ (with respect to $\nu$ ) satisfy

$$
\mathbb{E}_{\nu}\left[C_{n}\right]=\Lambda_{w}^{\prime}(s, w) n+O(1), \quad \mathbb{V}_{\nu}\left[C_{n}\right]=\Lambda_{w^{2}}^{\prime \prime}(s, w) n+O(1) .
$$

Suppose furthermore that $\Lambda_{w^{2}}^{\prime \prime}(s, w)$ is not zero. Then the costs $C_{n}$ asymptotically follow a Gaussian law (with respect to $\nu$ ).

Proof. The moment generating function of $C_{n}$ with respect to $\nu$,

$$
\mathbb{E}_{\nu}\left[\exp \left(u C_{n}\right)\right]:=\sum_{\mathbf{m} \in \mathcal{M}^{n}} \exp [u c(\mathbf{m})] \nu\left(I_{\mathbf{m}}\right)
$$

also equals (by $(5.2))$

$$
\lambda(s, w)^{-n} \sum_{\mathbf{m} \in \mathcal{M}^{n}} \exp [u c(\mathbf{m})] \int_{I} \mathbf{H}_{\mathbf{m}, s, w}[g] d \nu_{s, w}=\lambda(s, w)^{-n} \int_{I} \mathbf{H}_{s, w+u}^{n}[g] d \nu_{s, w}
$$

Since $w<0$, there exists a (complex) neighbourhood of $u=0$ for which $\Re(w+u)<0$. Then the quasi-power expression for the operator $\mathbf{H}_{s, w+u}^{n}$ given in (4.7) entails a quasi-power expression for the moment generating function,

$$
\mathbb{E}\left[\exp \left(u C_{n}\right)\right]=\left(\frac{\lambda(s, w+u)}{\lambda(s, w)}\right)^{n} a_{s, w}(u)\left(1+O\left(\alpha^{n}\right)\right)
$$

where $a_{s, w}(u)$ is bounded and $\alpha$ is related to the spectral gap of the operator $\mathbf{H}_{s, w+u}$. If $w+u$ belongs to a compact set included in $\Re(w)<0$, we can choose $|\alpha| \leq \alpha_{0}$ uniformly in $u$. We then apply the following quasi-powers theorem due to Hwang [25-27].

QuAsI-POWERS THEOREM. Assume that the moment generating functions for a sequence of functions $C_{n}$ are analytic in a complex neighbourhood 
$\mathcal{W}$ of $u=0$, and satisfy

$$
\mathbb{E}\left[\exp \left(u C_{n}\right)\right]=\exp \left[\beta_{n} U(u)+V(u)\right]\left(1+O\left(\kappa_{n}^{-1}\right)\right),
$$

with $\beta_{n}, \kappa_{n} \rightarrow \infty$ as $n \rightarrow \infty$, and $U(u), V(u)$ analytic on $\mathcal{W}$. Then the mean and variance satisfy

$\mathbb{E}\left[C_{n}\right]=U^{\prime}(0) \beta_{n}+V^{\prime}(0)+O\left(\kappa_{n}^{-1}\right), \quad \mathbb{V}\left[C_{n}\right]=U^{\prime \prime}(0) \beta_{n}+V^{\prime \prime}(0)+O\left(\kappa_{n}^{-1}\right)$.

Furthermore, if $U^{\prime \prime}(0) \neq 0$, the distribution of $C_{n}$ is asymptotically Gaussian, with speed of convergence $O\left(\kappa_{n}^{-1}+\beta_{n}^{-1 / 2}\right)$,

$$
\mathbb{P}_{\nu}\left[x \mid \frac{C_{n}(x)-U^{\prime}(0) n}{\sqrt{U^{\prime \prime}(0) n}} \leq Y\right]=\frac{1}{\sqrt{2 \pi}} \int_{-\infty}^{Y} e^{-y^{2} / 2} d y+O\left(\kappa_{n}^{-1}+\beta_{n}^{-1 / 2}\right) .
$$

The hypotheses of this theorem are fulfilled here with $\beta_{n}:=n$. The function $U_{[s, w]}$ defined by $U_{[s, w]}(u):=\Lambda(s, w+u)-\Lambda(s, w)$ is analytic around $u=0$ because $w \mapsto \Lambda(s, w)$ is analytic around $w<0$. At $u=0$, the first derivative $U_{[s, w]}^{\prime}(0)$ equals $\Lambda_{w}^{\prime}(s, w)$, and the second derivative $U_{[s, w]}^{\prime \prime}(0)$ equals $\Lambda_{w^{2}}^{\prime \prime}(s, w)$. If this quantity is not zero, then the variables $C_{n}$ follow an asymptotic Gaussian law.

Lemma 14. If the cost $c$ is not constant, then $\Lambda_{w^{2}}^{\prime \prime}$ is never zero when $(s, w)$ belongs to $\mathcal{S}_{1}$.

Proof. This proof is a close adaptation of a proof due to Broise [8]. However, our context is slightly different since the cost $c$ of interest does not belong to the reference function space $\mathcal{C}^{1}$. Moreover, the proof of Broise is only done for $(s, w)=(1,0)$.

We consider here the particular case where $\nu$ is defined by $d \nu:=f_{s, w} d \nu_{s, w}$ where $f_{s, w}$ is the dominant eigenfunction of $\mathbf{H}_{s, w}$ [we recall that it is strictly positive]. We consider the centred version of $c$, i.e.,

$$
\bar{c}:=c-\int_{I} c(t) d \nu(t), \quad \text { for which } \int_{I} \bar{c}(t) d \nu(t)=0,
$$

and denote by $\bar{C}_{n}$ the centred version of $C_{n}$, i.e.,

$$
\bar{C}_{n}:=\sum_{i=0}^{n-1} \bar{c} \circ T^{i}
$$

We denote by $\mathbf{L}_{s, w}, \mathbf{M}_{s, w}$ the normalized operators defined as

$$
\mathbf{L}_{s, w}[g]:=\frac{1}{\lambda(s, w) f_{s, w}} \mathbf{H}_{s, w}\left[g \cdot f_{s, w}\right], \quad \mathbf{M}_{s, w}:=\frac{1}{\lambda(s, w) f_{s, w}} \mathbf{N}_{s, w}\left[g \cdot f_{s, w}\right] .
$$

[Here, $\mathbf{N}_{s, w}$ is defined in (4.6).] Then $\mathbf{L}_{s, w}$ has a dominant eigenvalue equal to 1 , with an eigenfunction constantly equal to 1 . The eigenmeasure invariant under $\mathbf{L}_{s, w}^{\star}$ is exactly $\nu$ defined by $d \nu:=f_{s, w} d \nu_{s, w}$. The operator $\mathbf{L}_{s, w}$ acts on $\mathcal{L}^{1}[\nu]$, and even if the cost $\bar{c}$ does not belong to $\mathcal{C}^{1}$, its transform $\mathbf{L}_{s, w}[\bar{c}]$ 
belongs to $\mathcal{C}^{1}$. Furthermore, since $\mathcal{C}^{1}$ is dense in $\mathcal{L}^{2}[\nu]$, and since $\mathcal{L}^{2}[\nu]$ is a subset of $\mathcal{L}^{1}[\nu]$, the operator $\mathbf{M}_{s, w}$ can be extended to $\mathcal{L}^{2}[\nu]$ and the following holds, for any $g, f \in \mathcal{L}^{2}[\nu]$ :

$$
\begin{gathered}
\mathbf{L}_{s, w}[g]=\left(\int_{I} g d \nu\right)+\mathbf{M}_{s, w}[g], \quad \mathbf{M}_{s, w}[1]=0, \quad \mathbf{L}_{s, w}[g \circ T]=g, \\
\int_{I} f \circ T \cdot g d \nu=\int_{I} f \mathbf{L}_{s, w}[g] d \nu, \quad \int_{I} \bar{c} \mathbf{L}_{s, w}[g] d \nu=\int_{I} \bar{c} \mathbf{M}_{s, w}[g] d \nu .
\end{gathered}
$$

We suppose that $\Lambda_{w^{2}}^{\prime \prime}(s, w)=0$, and we wish to prove that $\bar{c}$ is zero. If $\Lambda_{w^{2}}^{\prime \prime}(s, w)=0$, then Lemma 13 proves that $\mathbb{V}_{\nu}\left[\bar{C}_{n}\right]=\mathbb{V}_{\nu}\left[C_{n}\right]$ is $O(1)$, so that $\bar{C}_{n}$ is uniformly bounded in $\mathcal{L}^{2}[\nu]$ by some constant $K$. For any $g \in \mathcal{C}^{1}$, the sequence

$$
R_{n}(g):=\int_{I} \bar{C}_{n} g d \nu=\sum_{k=0}^{n-1} \int_{I} \bar{c} \mathbf{L}_{s, w}^{k}[g] d \nu=\sum_{k=0}^{n-1} \int_{I} \bar{c} \mathbf{M}_{s, w}^{k}[g] d \nu
$$

is well-defined, satisfies $\left|R_{n}(g)\right| \leq K\|g\|_{2}$ and has a limit $R(g)$ which satisfies

$$
R(g):=\lim _{n \rightarrow \infty} R_{n}(g)=\int_{I} \bar{c}\left(I-\mathbf{M}_{s, w}\right)^{-1}[g] d \nu, \quad|R(g)| \leq K\|g\|_{2} .
$$

Since $\mathcal{C}^{1}$ is dense in $\mathcal{L}^{2}[\nu]$, the sequence $\bar{C}_{n}$ is weakly convergent in $\mathcal{L}^{2}[\nu]$, and $\bar{C}$ denotes its weak limit which belongs to $\mathcal{L}^{2}[\nu]$. For any sequence $g_{n} \in \mathcal{C}^{1}$ which converges in $\mathcal{L}^{2}[\nu]$ to $g \in \mathcal{L}^{2}$, one has

$$
\int_{I} \bar{C} g d \nu=\lim _{n \rightarrow \infty} \int \bar{c}\left(I-\mathbf{M}_{s, w}\right)^{-1}\left[g_{n}\right] d \nu=\lim _{n \rightarrow \infty} \int_{I} \bar{C} g_{n} d \nu .
$$

We first prove that $\bar{c}=\bar{C}-\bar{C} \circ T$ in $\mathcal{L}^{2}[\nu]$, or equivalently, $\int_{I} \bar{C} \circ T \cdot g d \nu$ $=\int_{I} \bar{C} g d \nu-\int_{I} \bar{c} g d \nu$ for any $g \in \mathcal{L}^{2}[\nu]$. Since

$$
\int_{I} \bar{C} \circ T \cdot g d \nu=\int_{I} \bar{C} \mathbf{L}_{s, w}[g] d \nu
$$

for any sequence $g_{n} \in \mathcal{C}^{1}$ which converges in $\mathcal{L}^{2}[\nu]$ to $g \in \mathcal{L}^{2}[\nu]$, one has

$$
\begin{aligned}
\int_{I} \bar{C} \circ T \cdot g d \nu & =\lim _{n \rightarrow \infty} \int_{I} \bar{c}\left(I-\mathbf{M}_{s, w}\right)^{-1} \circ \mathbf{L}_{s, w}\left[g_{n}\right] d \nu \\
& =\lim _{n \rightarrow \infty} \int_{I} \bar{c}\left(I-\mathbf{M}_{s, w}\right)^{-1} \circ \mathbf{M}_{s, w}\left[g_{n}\right] d \nu \\
& =\lim _{n \rightarrow \infty} \int_{I} \bar{c}\left[\left(I-\mathbf{M}_{s, w}\right)^{-1}-I\right]\left[g_{n}\right] d \nu .
\end{aligned}
$$

Since both limits

$$
\lim _{n \rightarrow \infty} \int_{I} \bar{C} g_{n} d \nu, \quad \lim _{n \rightarrow \infty} \int_{I} \bar{c} g_{n} d \nu
$$

exist and equal $\int_{I} \bar{C} g d \nu$ and $\int_{I} \bar{c} g d \nu$ respectively, the equality is proven. 
Now, we prove that $\bar{C}$ belongs to $\mathcal{C}^{1}$. First,

$$
\mathbf{L}_{s, w}[\bar{C} \circ T]=\mathbf{L}_{s, w}[\bar{C}]-\mathbf{L}_{s, w}[\bar{c}]=\mathbf{M}_{s, w}[\bar{C}]-\mathbf{M}_{s, w}[\bar{c}] .
$$

[The last equality holds since $\int_{I} \bar{C} d \nu=\int_{I} \bar{c} d \nu=0$.] On the other hand, by the definitions of $T$ and $\mathbf{L}_{s, w}$, one has $\mathbf{L}_{s, w}[\bar{C} \circ T]=\bar{C}$ and finally

$$
\bar{C}=-\left(I-\mathbf{M}_{s, w}\right)^{-1} \circ \mathbf{M}_{s, w}[\bar{c}] .
$$

Note that the function $\mathbf{M}_{s, w}[\bar{c}]=\mathbf{L}_{s, w}[\bar{c}]$ belongs to $\mathcal{C}^{1}$, so that $\left(I-\mathbf{M}_{s, w}\right)^{-1}$ 。 $\mathbf{M}_{s, w}[\bar{c}]$ is well-defined and $\bar{C}$ belongs to $\mathcal{C}^{1}$.

Now, the equality $\bar{c}=\bar{C}-\bar{C} \circ T$ holds at any point $x$ where $\bar{c}$ and $\bar{C} \circ T$ are well-defined, i.e., inside the open fundamental intervals of depth 1 . Consider the fixed point $h^{*}$ of each inverse branch $h$ of $T$, which belongs to the interior of the fundamental interval $I_{h}$. Then $\bar{c}\left(h^{*}\right)=0$ for any $h \in \mathcal{H}$, proving that $\bar{c}$ is zero, and $c$ is constant.

End of proof of Proposition 3. Now, when $w=\eta(M, s)$ is given by Lemma 11, the coefficient of the dominant term in the mean of $C_{n}$ is $\Lambda_{w}^{\prime}(s, \eta(M, s))=M$. Then, thanks to Lemmas 13 and 14 , the probability $\Pi_{n}$ of Lemma 12 can be approximated (when $n$ goes to $\infty$ ) by the corresponding probability of the Gaussian distribution

$$
\Pi_{n}=d(s)+O(1 / \sqrt{n}) \quad \text { with } \quad d(s):=\frac{1}{\sqrt{2 \pi}} \int_{\sqrt{-1 / U_{[s, w]}^{\prime \prime}(0)}}^{0} e^{-t^{2} / 2} d t .
$$

For $n$ large enough, one has $\Pi_{n}>d(s) / 2$, and one obtains, by (5.3),

$$
D_{n}(M, s) \geq b(s, w) \frac{d(s)}{2} e^{\eta \sqrt{n}}\left(e^{-\eta M} \lambda(s, \eta)\right)^{n},
$$

and finally, by the definition of $\alpha_{M}(s)$ given in Lemma 11,

$$
\liminf _{n \rightarrow \infty}\left[D_{n}(M, s)\right]^{1 / n} \geq \alpha_{M}(s) .
$$

Now, the other inequality of Lemma 12 is also true for $w=\eta(M, s)$, and

$$
\limsup _{n \rightarrow \infty}\left[D_{n}(M, s)\right]^{1 / n} \leq \alpha_{M}(s) .
$$

Finally, the sequence $\left[D_{n}(M, s)\right]^{1 / n}$ tends to $\alpha_{M}(s)$.

5.3. Properties of $\alpha_{M}$. The next lemma describes the main properties of the function $\alpha_{M}(s)$.

Lemma 15. Consider a triple of $\mathcal{G} \mathcal{L G}$-type. Let $M \in] \gamma(c), \mu(c)[$, and consider the neighbourhood $\mathcal{V}_{M}$ defined in Section 5.1. The map $\alpha_{M}: \mathcal{V}_{M} \rightarrow$ $\mathbb{R}^{+}$is strictly decreasing, and there exists a unique value $s=s_{M} \in \mathcal{V}_{M}$ for which $\alpha_{M}(s)=1$. 
Proof. Since $\Lambda_{w^{2}}^{\prime \prime}(s, w) \neq 0$, the implicit function theorem applies to the equation $\Lambda_{w}^{\prime}(s, w)=M$, and the solution $w=\eta(M, s)$ of this equation is analytic with respect to $s$. The same holds for $s \mapsto \alpha_{M}(s)$, since $\alpha_{M}(s)$ is the value of $w \mapsto \exp [-M w] \lambda(s, w)$ at $w=\eta(M, s)$. Proposition 3 shows that

$$
\alpha_{M}(s)=\lim _{n \rightarrow \infty}\left[A_{n}(M, s)\right]^{1 / n} \quad \text { with } \quad A_{n}(M, s)=\sum_{\mathbf{m} \in \mathcal{A}_{n}(M)} p_{\mathbf{m}}^{s} .
$$

The contraction property entails that $p_{\mathbf{m}} \leq \delta^{n}$ for any $\mathbf{m} \in \mathcal{M}^{n}$, so that

$$
A_{n}(M, s+\varrho) \sum_{\mathbf{m} \in \mathcal{A}_{n}(M)} p_{\mathbf{m}}^{s+\varrho} \leq \delta^{\varrho n} A_{n}(M, s) \quad \forall \varrho>0,
$$

and $\alpha_{M}(s+\varrho) \leq \delta^{\varrho} \alpha_{M}(s)<\alpha_{M}(s)$. Thus, $\alpha_{M}$ is strictly decreasing.

We now consider the two cases described in Section 5.1. If $\mathcal{V}_{M}=[0,1]$, one has for $s=0$, and $M \geq \gamma(c)$,

$$
A_{n}(M, 0)=\operatorname{card} \mathcal{A}_{n}(M) \geq 1 \quad \text { so that } \quad \alpha_{M}(0) \geq 1 .
$$

If $\left.\left.\mathcal{V}_{M}=\right] t_{M}, 1\right]$ with $t_{M}<1$, the value $\eta\left(t_{M}, M\right)$ equals 0 and

$$
\alpha_{M}\left(t_{M}\right)=\lambda\left(t_{M}, 0\right)=\lambda\left(t_{M}\right)>1 .
$$

For $s=1$, the family of fundamental intervals is a fundamental cover of ] $0,1[$, and

$$
A_{n}(M, 1) \leq \sum_{\mathbf{m} \in \mathcal{M}^{n}} p_{\mathbf{m}}=1 .
$$

Finally, $\alpha_{M}(0) \geq 1$ or $\alpha_{M}\left(t_{M}\right)>1$ whereas $\alpha_{M}(1) \leq 1$. Since $\alpha_{M}$ is strictly decreasing and continuous, there exists a unique $s=s_{M} \in \mathcal{V}_{M}$ for which $\alpha_{M}(s)=1$.

5.4. End of proof of Theorem 1. Finally, our Theorem 1 characterizes the Hausdorff dimension of $F_{M}$. It is the first main result of this paper.

Theorem 1. Consider the set $F_{M}$ relative to a triple $(I, T, c)$ of $\mathcal{G} \mathcal{L G}$ type. Denote by $\mathbf{H}_{s, w}$ the weighted operator relative to $(I, T, c)$ defined by

$$
\mathbf{H}_{s, w}[f]:=\sum_{m \in \mathcal{M}} \exp [w c(m)] \cdot\left|h_{m}^{\prime}\right|^{s} \cdot f \circ h_{m},
$$

and by $\Lambda(s, w)$ the logarithm of its dominant eigenvalue when $\mathbf{H}_{s, w}$ acts on $\mathcal{C}^{1}(I)$. Let $\gamma(c)$ be the minimal value of the cost $c$, and $\mu(c)$ be the stationary average of $c($ see (1.6)). Then, for any $\gamma(c)<M<\mu(c)$, there exists a unique pair $\left.\left(s_{M}, w_{M}\right) \in[0,1] \times\right]-\infty, 0[$ for which

$$
(\mathcal{S}): \quad \Lambda(s, w)=M w, \quad \frac{\partial}{\partial w} \Lambda(s, w)=M,
$$


and $s_{M}$ is the Hausdorff dimension of $F_{M}$. Moreover, the functions $M \mapsto s_{M}$ and $M \mapsto w_{M}$ are analytic at any point $\left.M \in\right] \gamma(c), \mu(c)[$.

Proof. It is based on Propositions 1 and 3, together with Lemma 15 and Corollary 1 . The real $w_{M}$ is just $w_{M}=\eta\left(M, s_{M}\right)$. Consider the determinant

$$
\begin{array}{rr}
\left|\begin{array}{cc}
\Lambda_{w}^{\prime}(s, w)-M & \Lambda_{w^{2}}^{\prime \prime}(s, w) \\
\Lambda_{s}^{\prime}(s, w) & \Lambda_{s^{2}}^{\prime \prime}(s, w)
\end{array}\right| & =\left(\Lambda_{w}^{\prime}(s, w)-M\right) \Lambda_{s^{2}}^{\prime \prime}(s, w)-\Lambda_{s}^{\prime}(s, w) \Lambda_{w^{2}}^{\prime \prime}(s, w) .
\end{array}
$$

On the curve defined by $(\mathcal{S})$, it reduces to its second term. Since $\Lambda_{s}^{\prime}(s, w)<0$, and $\Lambda_{w^{2}}^{\prime \prime}(s, w)>0$, this determinant is never zero. Hence, the implicit function theorem entails the analyticity of $M \mapsto s_{M}$ and $M \mapsto w_{M}$.

5.5. Boundary triples: general facts about $s_{M}$. We now focus on the boundary triples $(I, T, c)$ for which the critical abscissa equals 1 . In this case, the Dirichlet series defined in (2.5) has abscissa of convergence $s=1$ and is divergent at $s=1$. Hence, by Lemma 10 , the average $\mu(c)=$ $\lim _{w \rightarrow 0^{-}} \Lambda_{w}^{\prime}(1, w)$ is infinite, so that $\Lambda_{w^{2}}^{\prime \prime}(1, w) \rightarrow+\infty$ as $w \rightarrow 0^{-}$.

We wish to describe the asymptotic behaviour of $\operatorname{dim} F_{M}$ when $M \rightarrow$ $+\infty$. In this case, $\left(s_{M}, w_{M}\right) \rightarrow(1,0)$. We let $z:=\exp (-M)$, together with $s(z):=s_{M}, w(z):=-w_{M}$, and we consider the case when $z \rightarrow 0^{+}$. Then the pair $\left(s_{M}, w_{M}\right)$ is a solution of system $(\mathcal{S})$ if and only if $(s(z), w(z))$ is a solution of

$$
(\overline{\mathcal{S}}): \quad \lambda(s,-w)=z^{w}, \quad \lambda_{w}^{\prime}(s,-w)=-\log z \cdot z^{w} .
$$

When $z$ varies in $] 0, \exp [-\gamma(c)][$, these systems define a parameterized curve denoted by $\mathcal{C}$ which is the set of points $(s(z), w(z))$. The maps $z \mapsto w(z)$, $z \mapsto s(z)$ are analytic for $z \in] 0, \exp [-\gamma(c)][$. When $z=0$, this is no longer true, and we wish to describe the curve for $z \rightarrow 0$. If $z \rightarrow 0$, then $(s(z), w(z))$ $\rightarrow(1,0)$, and the first relation of (5.7) shows that $w(z) \log z \rightarrow 0$. The following lemma describes the behaviour of $s(z)-1$.

Lemma 16. Consider a boundary triple. For $z \rightarrow 0^{+}$, the behaviours of $s^{\prime}(z)$ and $w(z)$ are related by

$$
s^{\prime}(z)=\frac{1}{\Lambda_{s}^{\prime}(s(z),-w(z))} \cdot \frac{w(z)}{z},
$$

so that

$$
|s(z)-1|=\left[\frac{1}{h} \int_{0}^{z} \frac{w(t)}{t} d t\right] \cdot\left[1+O\left(\Lambda_{s}^{\prime}(s(z),-w(z))+h\right)\right] \quad \text { as } z \rightarrow 0^{+},
$$

where $h=-\Lambda_{s}^{\prime}(1,0)$ is the entropy of the dynamical system $(I, T)$. 
Proof. Differentiating the relation $\lambda(s,-w)=z^{w}$ with respect to $z$ gives

$$
\lambda_{s}^{\prime}(s,-w) s^{\prime}(z)-\lambda_{w}^{\prime}(s,-w) w^{\prime}(z)=z^{w}\left(w^{\prime}(z) \log z+\frac{w(z)}{z}\right) .
$$

Then the first and second relations of $(\overline{\mathcal{S}})$ entail

$$
\lambda_{s}^{\prime}(s,-w) s^{\prime}(z)=\lambda(s,-w) \frac{w(z)}{z} .
$$

If $z \rightarrow 0$, then $s(z)$ tends to $1, w(z)$ tends to 0 , and

$$
|s(z)-1|=-\int_{0}^{z} \frac{w(t)}{t} \cdot \frac{1}{\Lambda_{s}^{\prime}(s(t),-w(t))} d t .
$$

Since the function $z \mapsto \Lambda_{s}^{\prime}(s(z),-w(z))$ is continuous at $z=0$, it tends to the negative of the entropy $h$.

\section{DIRICHLET BOUNDARY TRIPLES: ASYMPTOTIC BEHAVIOUR OF $s_{M}$}

Here we introduce a subclass of boundary triples, the Dirichlet boundary triples. Then we state our Theorem 2 more precisely. The end of the section is devoted to proving this theorem, first in the general case. We then come back to our main motivation: the Euclidean dynamical system with the cost $c(m)=m$.

Consider a triple of $\mathcal{G} \mathcal{L G}$-type formed by a dynamical system and a cost $c$. Denote by $f_{1}$ the stationary density of $(I, T)$. When $(s, w)$ is near $(1,0)$, we shall deal with an approximation of $\lambda(s, w)$ which will be denoted by $\bar{\lambda}(s, w)$. Instead of the integrals $I_{m}(s, w), J_{m}(s, w)$ defined in (4.12), (4.13) which appear in the expression of $\lambda(s, w)$ and its derivatives, we will use the integrals

$$
I_{m}(s):=\int_{I}\left|h_{m}^{\prime}(t)\right|^{s} f_{1}(t) d t, \quad J_{m}(s):=\int_{I} \log \left|h_{m}^{\prime}(t)\right| \cdot\left|h_{m}^{\prime}(t)\right|^{s} f_{1}(t) d t,
$$

and consider

$$
\bar{\lambda}(s, w):=\sum_{m=1}^{\infty} \exp [w c(m)] I_{m}(s) .
$$

Now, the expressions that define $\bar{\lambda}$ and its derivatives are harmonic sums (with respect to $w$ ), i.e., sums of the form

$$
S_{s}(w)=\sum_{m \in \mathcal{M}} a_{m}(s) f\left(b_{m} w\right)
$$

The Mellin transform of such a harmonic sum factorizes as

$$
S_{s}^{\star}(u)=\left(\sum_{m} \frac{1}{b_{m}^{u}} a_{m}(s)\right) f^{\star}(u),
$$


and this is why the Mellin transform is so useful in this case. Here, the Mellin transforms of the functions $w \mapsto \bar{\lambda}(s, w), w \mapsto \bar{\lambda}_{w}^{\prime}(s, w), w \mapsto \bar{\lambda}_{s}^{\prime}(s, w)$ involve the two Dirichlet series

$$
I(s, u):=\sum_{m \in \mathcal{M}} \frac{1}{c(m)^{u}} I_{m}(s), \quad J(s, u):=\sum_{m \in \mathcal{M}} \frac{1}{c(m)^{u}} J_{m}(s) .
$$

For any $\left.s \in] \sigma_{0}, 1\right]$, the series $I_{s}(u):=I(s, u)$ and $J_{s}(u):=J(s, u)$ have convergence abscissa equal to some $u(s)$. The function $s \mapsto u(s)$ is decreasing, and the critical abscissa $\sigma_{1}$ is defined via the equation $u(s)=-1$. Then the boundary triples are those for which $u(1)=-1$.

6.1. Dirichlet boundary triples. In order to easily deal with the Mellin transform which will be a very powerful tool, we introduce more precise conditions on the Dirichlet series $I(s, u), J(s, u)$. For a general treatment of Mellin transforms in similar frameworks, see [16].

Definition 6. Associate to a boundary triple of $\mathcal{G} \mathcal{L G}$-type (formed by a dynamical system and a cost $c$ ) the two Dirichlet series $I(s, u), J(s, u)$. Denote by $u(s)$ the common convergence abscissa of the series $I_{s}(u):=$ $I(s, u), J_{s}(u):=J(s, u)$.

The triple is a Dirichlet boundary triple iff the following holds:

(a) for any $\left.s \in] \sigma_{0}, 1\right]$, there exists a strip $v(s) \leq \Re u \leq u(s)$ whose width $u(s)-v(s)$ is at least $\varepsilon$ (for some $\varepsilon>0$ which does not depend on $s$ ) and inside which $I_{s}, J_{s}$ have a unique pole (simple for $I_{s}$, double for $\left.J_{s}\right)$, located at $u=u(s)$, with expansions of the form

$$
I_{s}(u)=\frac{C(s)}{u-u(s)}+K(s)+o(1), \quad J_{s}(u)=\frac{D(s)}{(u-u(s))^{2}}+o(1), \quad u \rightarrow u(s),
$$

which define $\mathcal{C}^{2}$ functions $C(s), D(s)$ of $s$. Let $C:=C(1)$ and $D:=$ $D(1)$. Moreover, $I_{s}, J_{s}$ are of polynomial growth in the strip $v(s) \leq$ $\Re u \leq u(s)$ (uniformly in $s$ ).

(b) The function $s \mapsto u(s)$ is decreasing, equals -1 at $s=1$, is $\mathcal{C}^{2}$ with a derivative equal to $-B$ at $s=1$.

(c) The functions $I(s,-1), J(s,-1)$ have a pole at $s=1$ and satisfy

$$
I(s,-1)=\frac{A}{s-1}+K_{1}+o(1), \quad J(s,-1)=-\frac{A}{(s-1)^{2}}+o(1), \quad s \rightarrow 1 .
$$

(d) $B A=C$ and $D=-B C$.

REMARK. Assertion (d) is quite natural: it is obtained by comparison of (a) and (c) when $(u, s)$ is near $(-1,1)$, provided that some "uniformity" holds in the expansion (a).

Main instances. These conditions are fulfilled for all the memoryless boundary Riemann sources $\mathcal{B R}(\alpha)$. In this case, the series $I$ and $J$ involve 
the $\zeta$ function $\zeta(s)$ and its derivative $\zeta^{\prime}(s)$ :

$$
I(s, u)=\frac{1}{\zeta(\alpha)^{s}} \zeta(\alpha s+u(\alpha-1)), \quad J(s, u)=\frac{\alpha}{\zeta(\alpha)^{s}} \zeta^{\prime}(\alpha s+u(\alpha-1)) .
$$

The function $u(s)$ is linear and equals

$$
u(s)=\frac{1-\alpha s}{\alpha-1} \quad \text { with } \quad B=\frac{\alpha}{\alpha-1} .
$$

By the properties of the zeta function, we can choose $v(s):=u(s)-1 / 2$, and thus $\varepsilon=1 / 2$. Note also that

$$
\begin{gathered}
C(s)=\frac{1}{(\alpha-1) \zeta(\alpha)^{s}}, \quad A=\frac{1}{\alpha \zeta(\alpha)}, \\
K(s)=\frac{\gamma}{\zeta(\alpha)^{s}}, \quad L=\frac{1}{\zeta(\alpha)}\left(\gamma-\frac{1}{\alpha} \frac{\log \zeta(\alpha)}{\zeta(\alpha)}\right) .
\end{gathered}
$$

We will see later that conditions of Definition 6 are also fulfilled for the Euclidean dynamical system with $c(m)=m$.

6.2. Statement of Theorem 2. In this general framework, we can provide a precise estimate of the difference $\left|s_{M}-1\right|$ when $M \rightarrow \infty$. We then focus on two particular cases: the boundary Riemann system and the boundary triple relative to the Euclidean dynamical system with cost $c(m)=m$.

Theorem 2. Consider a Dirichlet boundary triple of $\mathcal{G} \mathcal{L G}$-type. Then the Hausdorff dimension of the set $F_{M}$ satisfies, as $M \rightarrow \infty$,

$$
\left|s_{M}-1\right|=\frac{C}{h} \exp \left[\frac{K}{C}-\gamma\right] e^{-M / C}\left[1+O\left(e^{-M \theta}\right)\right] \quad \text { with any } \theta<\frac{1}{C} .
$$

Here, $\gamma$ is the Euler constant, $h$ is the entropy, $C$ is the residue of $I_{1}(u)$ at $u=-1$, and $K$ is a constant which can be expressed through the constants which appear in Definition 6.

For the boundary Riemann triple $\mathcal{B R}(\alpha)$, for any $\theta<(\alpha-1) \zeta(\alpha)$ one has

$$
\left|s_{M}-1\right|=\frac{e^{\gamma(\alpha-2)}}{(\alpha-1) \zeta(\alpha) h(\alpha)} e^{-M(\alpha-1) \zeta(\alpha)}\left[1+O\left(e^{-M \theta}\right)\right]
$$

with

$$
h(\alpha)=\alpha \frac{\zeta^{\prime}(\alpha)}{\zeta(\alpha)}-\log \zeta(\alpha)
$$

For the Euclidean dynamical system with $c(m)=m$, for any $\theta<2$ one has

$$
\left|s_{M}-1\right|=\frac{6}{\pi^{2}} e^{-1-\gamma} 2^{-M}\left[1+O\left(\theta^{-M}\right)\right] .
$$

Plan of the proof. We first obtain, in Lemma 17, an upper bound for $\left|s_{M}-1\right|$. We then introduce a quantity $\bar{\lambda}(s, w)$ which will provide a good approximation of the dominant eigenvalue (Lemma 18) and will be amenable 
to Mellin analysis (Lemma 19). With these three main results, we prove that the curve defined by the system $(\overline{\mathcal{S}})$ lies inside a suitable domain of the plane (Lemma 20). This ends the proof of the general case. We then come back to the two particular cases.

6.3. An upper bound for $\left|s_{M}-1\right|$. The comparison between the sets $F_{M}$ and the sets $E_{K}$ defined as

$$
E_{K}:=\left\{x \in[0,1] ; m_{i}(x) \leq K, \forall i \geq 1\right\}
$$

provides an upper bound for $\left|s_{M}-1\right|$.

Lemma 17. For any Dirichlet boundary triple, $(s(z)-1) \log z$ is bounded when $z \rightarrow 0$.

Proof. The set $F_{M}$ contains the reals whose digits $m$ satisfy $c(m) \leq M$. The relation $c(m) \leq M$ is equivalent to $m \leq K_{M}$ (for some constant $K_{M}$ which depends on $M$ ), so that $F_{M}$ contains the set $E_{K_{M}}$. It is then sufficient to estimate the Hausdorff dimension $\tau_{K}$ of $E_{K}$. Here, we give an alternative proof to a weak version of the result of Hensley in the continued fraction context [21], namely $\left|\tau_{K}-1\right|=O(1 / K)$.

We recall that the Hausdorff dimension $\tau_{K}$ is characterized through the constrained operator

$$
\mathbf{H}_{[K], s}[f]:=\sum_{m \leq K}\left|h_{m}^{\prime}\right|^{s} \cdot f \circ h_{m},
$$

which is a particular case of the operator $\mathbf{H}_{\mathcal{A}, s}$ defined in (1.3). This constrained operator can be viewed as a perturbation of the plain operator $\mathbf{H}_{s}$ defined in (1.2). In particular, $\mathbf{H}_{[K], 1}$ is a perturbation of $\mathbf{H}:=\mathbf{H}_{1}$ which is a density transformer, with a dominant eigenfunction $f_{1}$ relative to $\lambda(1)=1$. The dominant spectral objects of $\mathbf{H}_{[K], s}$ are denoted by $\lambda_{[K]}(s), f_{[K], s}$. The Hausdorff dimension $\tau_{K}$ satisfies the equation $\lambda_{[K]}\left(\tau_{K}\right)=1$.

We first relate the difference $1-\lambda_{[K]}(1)$ of eigenvalues to the sup norm $\left\|\mathbf{H}-\mathbf{H}_{[K], 1}\right\|_{\infty}$ of the difference of the associated operators. By the two relations

$$
\mathbf{H}_{[K], 1}\left[f_{[K], 1}\right]=\lambda_{[K]}(1) f_{[K], 1}, \quad \mathbf{H}\left[f_{1}\right]=\lambda(1) f_{1}=f_{1},
$$

the following equality holds:

$$
\left(1-\lambda_{[K]}(1)\right) f_{[K], 1}+\left[f_{1}-f_{[K], 1}\right]=\left(\mathbf{H}-\mathbf{H}_{[K], 1}\right)\left[f_{[K], 1}\right]+\mathbf{H}\left[f_{1}-f_{[K], 1}\right] .
$$

We use the same arguments as in Lemma 9 , and consider the integral with respect to the Lebesgue measure. Since the plain operator $\mathbf{H}$ is a density transformer, it satisfies

$$
\int_{I} \mathbf{H}\left[f_{1}-f_{[K], 1}\right](t) d t=\int_{I}\left[f_{1}(t)-f_{[K], 1}(t)\right] d t
$$


which provides the equality

$$
\left(1-\lambda_{[K]}(1)\right) \int_{I} f_{[K], 1}(t) d t=\int_{I}\left(\mathbf{H}-\mathbf{H}_{[K], 1}\right)\left[f_{[K], 1]}\right](t) d t .
$$

From perturbation theory [30], the functions $f_{[K], 1}$ are positive and uniformly bounded from above and below for $K$ sufficiently large. The same holds for the integrals $\int f_{[K], 1}(t) d t$, and finally,

$$
1-\lambda_{[K]}(1) \leq A\left\|\mathbf{H}-\mathbf{H}_{[K], 1}\right\|_{\infty} \leq B \sum_{m \geq K} p_{m},
$$

where $A$ is some constant and $B:=A L$ involves the distortion constant $L$. The relation between $K_{M}$ and $M$ now implies that

$$
1-\lambda_{\left[K_{M}\right]}(1) \leq B G(M) \text { with } \quad G(x):=\sum_{m ; c(m) \geq x} p_{m} .
$$

The Mellin transform of the function $G$ is exactly $I(1, u)$ which has a pole at $u=1$. Then $G(x)$ is $\Theta(1 / x)$ as $x \rightarrow \infty$, and

$$
1-\lambda_{\left[K_{M}\right]}(1)=O(1 / M) \text {. }
$$

On the other hand, the mean value theorem entails that

$$
\lambda_{[K]}(1)-1=\lambda_{[K]}(1)-\lambda_{[K]}\left(\tau_{K}\right)=\left(1-\tau_{K}\right) \lambda_{[K]}^{\prime}\left(\bar{\tau}_{K}\right)
$$

where $\left.\bar{\tau}_{K} \in\right] \tau_{K}, 1\left[\right.$. Finally, if $M \rightarrow \infty$, then $K_{M}$ also tends to $\infty$, and $\left|\lambda_{K_{M}}^{\prime}\left(\bar{\tau}_{K_{M}}\right)\right|$ tends to the entropy $h$. From (6.2) and (6.3), one obtains

$$
\left|s_{M}-1\right| \leq\left|\tau_{K_{M}}-1\right| \leq C / M \text { for some constant } C \text {. }
$$

6.4. Relations between $\lambda$ and its approximation $\bar{\lambda}$. We recall that we work with an approximation of $\lambda(s, w)$, denoted by $\bar{\lambda}(s, w)$ :

$$
\bar{\lambda}(s, w):=\sum_{m=1}^{\infty} \exp [w c(m)] I_{m}(s)
$$

which involves integrals $I_{m}(s)$ defined in (6.1). The next lemma proves that $\bar{\lambda}$ is indeed an approximation of $\lambda$.

LEMMA 18. When $(s, w) \rightarrow(1,0)$, the following holds:

(a) $\bar{\lambda}_{w}^{\prime}(s, w) / \lambda_{w}^{\prime}(s, w)$ has a lower bound and an upper bound.

(b) $\left|\bar{\lambda}_{w}^{\prime}(s, w)-\lambda_{w}^{\prime}(s, w)\right|=\lambda(s, w) O\left(|s-1|+w\left|\bar{\lambda}_{w}^{\prime}(s, w)\right|\right)$.

Proof. (a) We know from Lemma 10 that the map $(s, w) \mapsto \mathbf{H}_{s, w}$ is continuous at $(1,0)$. Then perturbation theory (with continuous perturbation, not analytic) is applied to the quasi-compact operator $\mathbf{H}_{s, w}$ near $(1,0)$, and proves that the dominant spectral objects are continuous at $(1,0)$.

Consider a compact neighbourhood $\mathcal{S}_{2}$ of $(1,0)$ in $\mathcal{S}_{1}$. Then the mapping $(s, w, t) \mapsto f_{s, w}(t)$ is continuous on the compact set $\mathcal{S}_{2} \times I$ and strictly 
positive, and it admits a lower bound $a$ and an upper bound $b$ strictly positive. We begin with the expressions

$$
\begin{aligned}
& \lambda_{w}^{\prime}(s, w)=\sum_{m=1}^{\infty} c(m) \exp [w c(m)] I_{m}(s, w), \\
& \bar{\lambda}_{w}^{\prime}(s, w)=\sum_{m=1}^{\infty} c(m) \exp [w c(m)] I_{m}(s),
\end{aligned}
$$

which involve the integrals $I_{m}(s, w)$ and $I_{m}(s)$ defined in (4.12) and (6.1). Then the distortion property entails that

$$
\frac{a}{L^{s}} \bar{\lambda}_{w}^{\prime}(s, w) \leq \lambda_{w}^{\prime}(s, w) \leq L^{s} b \bar{\lambda}_{w}^{\prime}(s, w)
$$

(b) Since

$$
\left|I_{m}(s)-I_{m}(s, w)\right|=p_{m}^{s}\left[O\left(\left\|f_{s, w}-f_{1}\right\|_{1}\right)+O\left(\left\|\nu_{s, w}-\nu_{1}\right\|_{1}\right)\right],
$$

it is sufficient to evaluate each term $\left\|f_{s, w}-f_{1}\right\|_{1}$ and $\left\|\nu_{s, w}-\nu_{1}\right\|_{1}$. Denote by $\widetilde{\mathbf{H}}_{s, w}$ the operator

$$
\widetilde{\mathbf{H}}_{s, w}:=\frac{1}{\lambda(s, w)} \mathbf{H}_{s, w} .
$$

The dominant eigenvalue $\widetilde{\lambda}(s, w)$ of $\widetilde{\mathbf{H}}_{s, w}$ is constant and equal to 1 , and $f_{s, w}$ is the dominant eigenfunction of $\widetilde{\mathbf{H}}_{s, w}$ relative to the eigenvalue 1. Differentiating the relation $\widetilde{\mathbf{H}}_{s, w}\left[f_{s, w}\right]=f_{s, w}$ with respect to $w$, and using the equality

$$
0=\frac{d}{d w} \widetilde{\lambda}(s, w)=\int_{I} \frac{d}{d w} \widetilde{\mathbf{H}}_{s, w}\left[f_{s, w}\right] d \nu_{s, w}(t)=0
$$

entails that

$$
\int_{I} g_{s, w}(t) d \nu_{s, w}(t)=0 \quad \text { with } \quad g_{s, w}:=\left(I-\widetilde{\mathbf{H}}_{s, w}\right)\left[\frac{d}{d w} f_{s, w}\right]=\left(\frac{d}{d w} \widetilde{\mathbf{H}}_{s, w}\right)\left[f_{s, w}\right] .
$$

Thus, the projection of $g_{s, w}$ on the dominant eigensubspace of $\widetilde{\mathbf{H}}_{s, w}$ equals 0 . Denote by $\widetilde{\mathbf{N}}_{s, w}$ the operator $\widetilde{\mathbf{N}}_{s, w}:=(1 / \lambda(s, w)) \mathbf{N}_{s, w}$ with $\mathbf{N}_{s, w}$ defined in (4.6). Then, for all $n \geq 1$, one has $\widetilde{\mathbf{H}}_{s, w}^{n}\left[g_{s, w}\right]=\widetilde{\mathbf{N}}_{s, w}^{n}\left[g_{s, w}\right]$. Now, the quasicompactness of $\widetilde{\mathbf{H}}_{s, w}$ proves that the series with general term $\widetilde{\mathbf{H}}_{s, w}^{n}\left[g_{s, w}\right]$ is convergent, with sum equal to $\frac{d}{d w} f_{s, w}$. Finally,

$$
\frac{d}{d w} f_{s, w}=\left(I-\widetilde{\mathbf{N}}_{s, w}\right)^{-1}\left[g_{s, w}\right]=\left(I-\widetilde{\mathbf{N}}_{s, w}\right)^{-1} \circ\left(\frac{d}{d w} \widetilde{\mathbf{H}}_{s, w}\right)\left[f_{s, w}\right] .
$$

Now,

$$
\left\|\frac{d}{d w} \widetilde{\mathbf{H}}_{s, w}\right\|_{1} \leq\left\|\frac{1}{\lambda(s, w)} \frac{d}{d w} \mathbf{H}_{s, w}\right\|_{1}+\left\|\frac{\lambda_{w}^{\prime}(s, w)}{\lambda(s, w)^{2}} \mathbf{H}_{s, w}\right\|_{1}
$$


By comparing to the memoryless approximate model and using arguments similar to those used in Lemma 10 and part (a) of the present lemma we are led to the estimate

$$
\left\|\frac{d}{d w} \widetilde{\mathbf{H}}_{s, w}\right\|_{1}=O\left(\left|\bar{\lambda}_{w}^{\prime}(s, w)\right|\right), \quad(s, w) \rightarrow(1,0) .
$$

On the other hand, the mapping $(s, w) \mapsto \widetilde{\mathbf{H}}_{s, w}$ is continuous at $(1,0)$, so that, by perturbation theory (with continuous perturbation), the norm of the operator $\left(I-\tilde{\mathbf{N}}_{s, w}\right)^{-1}$, and the function $f_{s, w}$, are bounded. Finally, one obtains

$$
\left\|\frac{d}{d w} f_{s, w}\right\|_{1}=O\left(\left|\bar{\lambda}_{w}^{\prime}(s, w)\right|\right), \quad(s, w) \rightarrow(1,0) .
$$

In the same vein, differentiating with respect to $s$ leads to

$$
\left\|\frac{d}{d s} f_{s, w}\right\|_{1}=O(1), \quad(s, w) \rightarrow(1,0) .
$$

Finally, we have proven that

$$
\left\|f_{s, w}-f_{1}\right\|_{1}=O(|s-1|)+O\left(w\left|\bar{\lambda}_{w}^{\prime}(s, w)\right|\right) \quad \text { as }(s, w) \rightarrow(1,0) .
$$

The proof is exactly the same for the dominant eigenmeasure $\nu_{s, w}$ of the dual operator $\mathbf{H}_{s, w}^{*}$.

6.5. Precise estimates of $\bar{\lambda}$ and its derivatives when $(s, w) \rightarrow$ $(1,0)$. The Dirichlet conditions allow us to use Mellin analysis, which provides precise estimates of $\bar{\lambda}$ and its derivatives when $(s, w) \rightarrow(1,0)$.

Lemma 19. For any Dirichlet boundary triple, the function $\bar{\lambda}(s, w)$ satisfies the following:

(a) If $(s, w) \rightarrow\left(1^{-}, 0^{+}\right)$, then for some $\varepsilon>0$ :

$$
\begin{gathered}
\bar{\lambda}(s,-w)-\lambda(s, 0) \\
=-w \frac{A}{s-1}-w \frac{C(s)}{u(s)+1} w^{-u(s)-1}+O\left(|s-1| w+w^{1+\varepsilon}\right) \\
\bar{\lambda}_{w}^{\prime}(s,-w)=\frac{1}{s-1}\left[A+C(s) \frac{s-1}{u(s)+1} w^{-u(s)-1}\right] \\
\quad+\left(K_{1}-C \gamma w^{-u(s)-1}\right)+O\left(|s-1|+w^{\varepsilon}\right) \\
\frac{1}{w}\left(\bar{\lambda}_{s}^{\prime}(s,-w)+h\right)=D(s) \frac{w^{-u(s)-1}}{(u(s)+1)^{2}}[(1+u(s)) \log w+1] \\
+\frac{A}{(s-1)^{2}}+O\left(|s-1|+w^{\varepsilon}\right) .
\end{gathered}
$$


(b) In particular, when $|s-1||\log w| \rightarrow 0$, one has, for some constant $K$ which is a function of the constants of Definition 6,

$$
\begin{aligned}
& \bar{\lambda}_{w}^{\prime}(s,-w)=-C \log w+(K-C \gamma)+O(s-1) \log ^{2} w+O\left(w^{\varepsilon}\right), \\
& \bar{\lambda}_{s}^{\prime}(s,-w)+h=O\left(w \log ^{2} w\right)+O\left(w^{1+\varepsilon}\right), \\
& \bar{\lambda}(s,-w)-1=O(|s-1| w \log w)+O\left(w^{1+\varepsilon}\right) .
\end{aligned}
$$

Proof. It uses Mellin transforms. The functions $\bar{\lambda}(s,-w), \bar{\lambda}_{w}^{\prime}(s,-w)$, $\bar{\lambda}_{s}^{\prime}(s,-w)$ are considered first as functions of $w$ and respectively denoted by $M_{s}(w), K_{s}(w), L_{s}(w)$. Recall that

$$
\begin{aligned}
& M_{s}(w)=\sum_{m \in \mathcal{M}} \exp [-c(m) w] I_{m}(s), \\
& K_{s}(w)=\sum_{m \in \mathcal{M}} c(m) \exp [-c(m) w] I_{m}(s), \\
& L_{s}(w)=\sum_{m \in \mathcal{M}} \exp [-c(m) w] J_{m}(s),
\end{aligned}
$$

where we assume $s$ is near 1 and $0<w<1$. The Mellin transforms of $M_{s}, K_{s}, L_{s}$, which are harmonic sums, are explicit and involve the three functions $\Gamma, I_{s}, J_{s}$ :

$$
M_{s}^{\star}(u)=\Gamma(u) I_{s}(u), \quad K_{s}^{\star}(u)=\Gamma(u) I_{s}(u-1), \quad L_{s}^{\star}(u)=\Gamma(u) J_{s}(u) .
$$

The transforms $M_{s}^{\star}, K_{s}^{\star}, L_{s}^{\star}$ have two kinds of poles: due to the $\Gamma$ function and due to the functions $I_{s}$ or $J_{s}$.

For $K_{s}^{\star}$, the poles are at the nearby points $u=0$ (due to $\Gamma$ ) and $u=$ $u(s)+1$ (due to $I_{s}$ ), and the existence strip of $K_{s}^{\star}$ is $\Re(u)>u(s)+1$. The Mellin inversion theorem yields, for any $D>u(s)+1$,

$$
K_{s}(w)=\frac{1}{2 i \pi} \int_{D-i \infty}^{D+i \infty} \Gamma(u) I(s, u-1) w^{-u} d u,
$$

and shifting the integration line to the left leads to

$$
\begin{aligned}
K_{s}(w)= & C(s) \Gamma(u(s)+1) w^{-u(s)-1}+I(s,-1) \\
& +\frac{1}{2 i \pi} \int_{-\varepsilon-i \infty}^{-\varepsilon+i \infty} \Gamma(u) I(s, u-1) w^{-u} d u .
\end{aligned}
$$

By property (a) of Definition 6, the remainder integral is $O\left(w^{\varepsilon}\right)$, uniformly with respect to $s$ in the stated range ( $s$ near 1 and $0<w<1)$. Consequently,

$$
K_{s}(w)=C(s) \Gamma(u(s)+1) w^{-u(s)-1}+I(s,-1)+O\left(w^{\varepsilon}\right) .
$$

For $M_{s}^{\star}$, the poles are at $u=0$, and at the nearby points $u=-1$ (due to $\Gamma$ ) and $u=u(s)$ (due to $I_{s}$ ). Applying the Mellin inversion theorem and 
shifting the relevant integration line to the left leads to

$$
M_{s}(w)-M_{s}(0)=-w I(s,-1)+C(s) w \cdot w^{-1-u(s)} \Gamma(u(s))+O\left(w^{1+\varepsilon}\right) .
$$

For $L_{s}^{\star}$, the poles are at $u=0$, and at the nearby points $u=-1$ (due to $\Gamma$ ) and $u=u(s)$ (due to $J_{s}$ ). Note that this last pole has order 2. Applying the Mellin inversion theorem and shifting the relevant integration line to the left leads to

$$
\begin{aligned}
L_{s}(w)-L_{s}(0)= & -w J(s,-1) \\
& +D(s) w \cdot w^{-1-u(s)}\left[-\Gamma(u(s)) \log w+\Gamma^{\prime}(u(s))\right]+O\left(w^{1+\varepsilon}\right) .
\end{aligned}
$$

Assume now that $s \rightarrow 1$. Then

$$
\begin{gathered}
\Gamma(u(s)+1)=\frac{1}{u(s)+1}-\gamma+O(s-1), \quad \Gamma(u(s))=-\frac{1}{u(s)+1}+O(1), \\
\Gamma^{\prime}(u(s))=\frac{1}{(u(s)+1)^{2}}+O(1) .
\end{gathered}
$$

Thus, by property (c) of Definition 6 , when $w$ and $s-1$ tend to 0 simultaneously, relations (6.5)-(6.7) hold.

If now $(s-1) \log w$ tends to 0 , then property (b) of Definition 6 entails that the factor $w^{-u(s)-1}-1$, omnipresent in all the relations, is equal to

$$
w^{-u(s)-1}-1=B(s-1) \log w+O\left(|s-1|^{2} \log ^{2} w\right),
$$

which easily implies part (b) of the lemma.

6.6. End of proof of Theorem 2. We now use Lemmas 16-19 in order to obtain a precise description of the curve $\mathcal{C}$ defined by the system $(\overline{\mathcal{S}})$ described in $(5.7)$.

Lemma 20. For any Dirichlet boundary triple, $(s(z)-1) \log w(z)$ tends to 0 as $z \rightarrow 0$. Moreover $\lambda_{w}^{\prime}(s, w)=O(\log w)$.

Proof. From the second equation of $(\overline{\mathcal{S}})$, together with Lemma 17, we deduce that $(s-1) \lambda_{w}^{\prime}(s, w)$ must be bounded for $z \rightarrow 0$. Now, Lemma 18 entails that $(s-1) \bar{\lambda}_{w}^{\prime}(s, w)$ must be bounded. And finally, by (6.6) of Lemma 19, we deduce that the term $w^{-u(s)-1}$ must be bounded for $z \rightarrow 0$. Since $u(s)+1>0$, this entails that $|s-1||\log w|$ is bounded, and finally $w^{-u(s)-1}-1=O(|s-1||\log w|)$. Thus, (6.6) proves that

$$
(s-1) \bar{\lambda}_{w}^{\prime}(s, w)=O\left(w^{-u(s)-1}-1\right)=O(|s-1||\log w|) .
$$

Lemma 18 entails that $\lambda_{w}^{\prime}(s, w)$ is also $O(|\log w|)$. Since $w(z) \log z \rightarrow 0$, the second relation of $(\overline{\mathcal{S}})$ proves that $|\log z| \leq A|\log w|$ for some constant $A$. And finally $z$ and $w$ are related by $w \leq z^{1 / A}$. Hence Lemma 16 shows that $|s-1|=O\left(z^{1 / A}\right)$ and finally $|s-1||\log z| \rightarrow 0$. 
Using again relation (6.6), the second equation of $(\overline{\mathcal{S}})$, and the last fact, we deduce that $(s-1) \lambda_{w}^{\prime}(s, w) \rightarrow 0$ as $z \rightarrow 0$. Then Lemma 18 entails that $(s-1) \bar{\lambda}_{w}^{\prime}(s, w) \rightarrow 0$. From (6.6), together with condition (d) of Definition 6, we deduce that $1-w^{-u(s)-1} \rightarrow 0$ as $z \rightarrow 0$. This entails that $|s-1||\log w|$ $\rightarrow 0$.

Now, we use again Lemma 18(b) to get

$$
\left|\bar{\lambda}_{w}^{\prime}(s, w)-\lambda_{w}^{\prime}(s, w)\right|=O(|s-1|)+O(w \log w),
$$

and Lemma 19(b) entails that

$$
\begin{aligned}
-\lambda_{w}^{\prime}(s,-w) & =C \log w-(K-C \gamma)+O(s-1) \log ^{2} w+O(w \log w) \\
& =\log z+O\left(w \log ^{2} z\right)
\end{aligned}
$$

Since both $w \log z$ and $|s-1| \log w$ tend to zero, this proves that $\left|\log z^{1 / C}\right|$ and $|\log w(z)|$ are equivalent, so that, for any $\varrho>1$, there exists a neighbourhood of $z=0$ on which $(1 / \varrho)|\log w(z)| \leq(1 / C)|\log z| \leq \varrho|\log w(z)|$. This means that $w^{\varrho} \leq z^{1 / C} \leq w^{1 / \varrho}$. Together with Lemma 16, this entails that

$$
|s-1|=O\left(z^{1 /(C \varrho)}\right)=O\left(w^{1 /\left(C \varrho^{2}\right)}\right)
$$

and both $w \log ^{2} z$ and $|s-1| \log ^{2} w$ are $O\left(z^{1 /(C \varrho)} \log ^{2} z\right)=O\left(z^{1 / C-\varepsilon}\right)$ for any $\varepsilon>0$. Returning to (6.8), we see that

$$
w=\exp \left[\frac{K}{C}-\gamma\right] z^{1 / C}+O\left(z^{2 / C-\varepsilon}\right), \quad z \rightarrow 0 .
$$

Finally, from Lemma 16 and the second and third relations of Lemma 19(b), we obtain

$$
|s-1|=\exp \left[\frac{K}{C}-\gamma\right] \frac{C}{h} z^{1 / C}\left[1+O\left(z^{(1 / C) \varepsilon}\right)\right]
$$

for any $\varepsilon>0$. Setting $z:=\exp (-M)$ ends the proof of Theorem 2 .

Note that the rôle played by the normalization constant $1 / C$ is due to the equality

$$
C=\lim \frac{1}{\log w} \lambda_{w}^{\prime}(s,-w) \quad\left(w \rightarrow 0^{-},(s-1) \log w \rightarrow 0\right) .
$$

6.7. A first particular case: the systems $\mathcal{B R}(\alpha)$. In this case, the constants $C$ and $K$ are

$$
C=C(1)=\frac{1}{(\alpha-1) \zeta(\alpha)}, \quad \frac{K}{C}=\frac{K(1)}{C(1)}=\gamma(\alpha-1) .
$$

6.8. A second particular case: the Euclidean system with the cost $c(m)=m$. We prove that the general framework of this section allows us to deal with our first motivation: the Euclidean system with the cost $c(m)=m$. 
LEMMA 21. The Euclidean dynamical system with the cost $c(m)=m$ is a Dirichlet boundary triple. The constant $C$ equals $1 / \log 2$, and the constant K of Lemma 19 (b) equals $-1 / \log 2$.

Proof. The integrals $I_{m}(s)$ are explicit

$$
I_{m}(s):=\int_{I}\left|h_{m}^{\prime}(t)\right|^{s} \cdot f_{1} \circ h_{m}(t) d t=\frac{1}{\log 2} \int_{1 /(m+1)}^{1 / m} \frac{t^{2 s-2}}{t+1} d t
$$

so that

$$
(\log 2) I_{m}(s)=\frac{1}{m^{2 s}}\left(1+O\left(\frac{1}{m}\right)\right)
$$

with $O$ uniform for $\Re s$ near 1 . Then the Dirichlet series $I(s, u)$ satisfies $(\log 2) I(s, u-1)=\zeta(2 s+u-1)+C(s, u-1) \quad$ with $\quad|C(\sigma, u)| \leq K|\zeta(2 \sigma+u)|$, and the unique pole of $I(s, u-1)$ near 0 is simple, equal to $u=2-2 s$, with residue $\operatorname{Res}[I(s, u-1), u=2-2 s]=1$. We deduce that the constant $C$ of Theorem 2 is $C=1 / \log 2$.

Since the Mellin transform of $\bar{\lambda}_{w}^{\prime}(s,-w)$ is $\Gamma(u) I(s, u-1)$, one has

$$
\bar{\lambda}_{w}^{\prime}(s,-w)=I(s,-1)+\Gamma(2-2 s) w^{2 s-2}+O(|s-1|)+O\left(w^{1 / 2}\right) .
$$

Now, when $s$ is near 1 , one has

$$
\begin{aligned}
(\log 2) I(s,-1) & =\zeta(2 s-1)+C(s,-1) \\
& =\frac{1}{2(s-1)}+\gamma+C(1,-1)+O(s-1), \\
\Gamma(2-2 s) & =-\frac{1}{2(s-1)}-\gamma+O(s-1) .
\end{aligned}
$$

When $|s-1||\log w|$ tends to 0 , $(\log 2) \cdot \bar{\lambda}_{w}^{\prime}(s,-w)=-\log w+C(1,-1)+O(s-1) \log ^{2} w=-(\log 2) \log z$ where

$$
C(1,-1):=\sum m\left[(\log 2) I_{m}(1)-1 / m^{2}\right]
$$

is a convergent series whose general term is

$$
m\left(F\left(\frac{1}{m}\right)-F\left(\frac{1}{m+1}\right)\right)-\frac{1}{m} \quad \text { with } \quad F(x)=\log (1+x) .
$$

Using Abel's transformation entails that $C(1,-1)$ equals $-1-\gamma$. Finally,

$$
w \sim K_{1} z^{\log 2} \text { with } K_{1}:=\exp C(1,-1)=\exp [-1-\gamma]
$$

which ends the proof of Theorem 2 .

\section{CONCLUSIONS AND OPEN PROBLEMS}

The two main results of this paper (Theorems 1 and 2) are of different nature. Theorem 1 is a general result which shows that the Hausdorff dimension of a wide class of sets can be characterized in terms of a solution of a 
differential system which involves the dominant eigenvalues of the weighted transfer operator. It deals with triples $(I, T, c)$ of large growth, and it is, in a sense, complementary to the multifractal result of [18] which is only obtained in the case of a triple of moderate growth. Is it possible to obtain our result in the $\mathcal{G} \mathcal{M G}$-setting? and the result of [18] in the $\mathcal{G} \mathcal{L} \mathcal{G}$-setting?

On the other hand, Theorem 2 deals with a particular framework and provides precise asymptotic estimates for the dimension of the set $F_{M}$, particularly in the continued fraction context. Theorem 2 proves that the characterization given in Theorem 1 is useful for effective computations, even for dynamical systems with memory. The main idea is to relate systems with memory to memoryless schemes which approximate them. It is clear that these "approximation" techniques are applicable to more general instances of dynamical systems.

Finally, Theorem 1 together with the results of Hanus, Mauldin and Urbański [18] poses an important question: Is it possible to describe a general framework where systematic computation of dimensions can be provided? In the case when the constraints deal with each digit in an independent way, there exist two algorithms. One was proposed by Hensley [22]; the other one, described by Daudé, Flajolet and Vallée [12], used in [41] and justified by Lhote [33], provides (in polynomial time) numerical values for the Hausdorff dimension. The present case is certainly more difficult, since now a system (of two equations) has to be solved, whereas there was previously a single equation to solve.

We precisely described the sets of reals whose continued fraction expansion has all its prefix digit averages less than $M$. To perform a precise analysis of the Euclidean subtractive algorithm (see $[42,43]$ ), one needs precise information on the set of rational numbers whose digits in the continued fraction expansion have an average less than $M$. This discrete problem is more difficult to solve than the present continuous one. In the case of "fast Euclidean algorithms", relative to costs of moderate growth, the weighted transfer operator $\mathbf{H}_{s, w}$ is analytic at the reference point $(s, w)=(1,0)$. Then Tauberian theorems or Perron's formula [42, 43, 3] allow a transfer "from continuous to discrete". Here, it does not seem possible to use directly these tools, due to the non-analyticity of $\mathbf{H}_{s, w}$ at $(1,0)$.

\section{References}

[1] V. Afraimovich and J. Urias, Dimension-like characteristics of invariant sets in dynamical systems, in: Dynamics and Randomness (Santiago 2000), Nonlinear Phenom. Complex Systems 7, Kluwer, 2002, 1-30.

[2] V. Baladi, Positive Transfer Operators and Decay of Correlations, World Sci., 2000. 
[3] V. Baladi and B. Vallée, Distributional analyses of Euclidean algorithms, in: Proc. ALENEX-ANALCO'04 (New Orleans), SIAM, 2004, 170-184.

[4] —, - Euclidean algorithms are Gaussian, J. Number Theory 110 (2005), 331-386.

[5] A. S. Besicovitch, Sets of fractional dimensions: On rational approximation to real numbers, J. London Math. Soc. 9 (1934), 126-131.

[6] - On the sum of digits of real numbers represented in the dyadic system, Math. Ann. 110 (1934), 321-330.

[7] P. Billingsley, Probability and Measure, Wiley, 1979.

[8] A. Broise, Transformations dilatantes de l'intervalle et théorèmes limites, Astérisque 238 (1996), 5-109.

[9] R. T. Bumby, Hausdorff dimension of sets arising in number theory, in: New York Number Theory Seminar, Lecture Notes in Math. 1135, Springer, New York, 1985, $1-8$.

[10] E. Cesaratto and B. Vallée, Reals with bounded digit averages, in: Proc. of the Colloquium on Mathematics and Computer Science: Algorithms, Trees, Combinatorics and Probability, M. Drmota et al. (eds.), Birkhäuser, 2004, 473-490.

[11] T. W. Cusick, Continuants with bounded digits, I, II, Mathematika 24 (1977), 166172, 25 (1978), 107-109.

[12] H. Daudé, P. Flajolet and B. Vallée, An average-case analysis of the Gaussian Algorithm for lattice reduction, Combin. Probab. Comput. 6 (1997), 397-433.

[13] H. Eggleston, The fractional dimension of a set defined by decimal properties, Quart. J. Math. Oxford Ser. 20 (1949), 31-36.

[14] K. Falconer, Fractal Geometry-Mathematical Foundations and Applications, Wiley, New York, 1990.

[15] —, Techniques in Fractal Geometry, Wiley, New York, 1997.

[16] P. Flajolet, X. Gourdon and P. Dumas, Mellin transforms and asymptotics: harmonic sums, Theoret. Comput. Sci. 144 (1995), 3-58.

[17] I. J. Good, The fractional dimensional theory of continued fractions, Math. Proc. Cambridge Philos. Soc. 37 (1941), 199-228.

[18] P. Hanus, D. Mauldin and M. Urbański, Thermodynamic formalism and multifractal analysis of conformal infinite iterated function system, Acta Math. Hungar. 96 (2002), 27-98.

[19] D. Hensley, The distribution of badly approximable rationals and continuants with bounded digits II, J. Number Theory 34 (1990), 293-334.

[20] - The Hausdorff dimensions of some continued fraction Cantor sets, ibid. 33 (1989), 182-198.

[21] - Continued fraction Cantor sets, Hausdorff dimension, and functional analysis, ibid. 40 (1992), 336-358.

[22] - A polynomial time algorithm for the Hausdorff dimension of a continued fraction Cantor set, ibid. 58 (1996), 9-45.

[23] - , The statistics of the continued fraction digit sum, Pacific J. Math. 192 (2000), $103-120$.

[24] K. E. Hirst, Continued fractions with sequences of partial quotients, Proc. Amer. Math. Soc. 38 (1973), 221-227.

[25] H.-K. Hwang, Théorèmes limite pour les structures combinatoires et les fonctions arithmétiques, $\mathrm{PhD}$ thesis, Ecole Polytechnique, 1994.

[26] —, Large deviations for combinatorial distributions: I, Central limit theorems, Ann. Appl. Probab. 6 (1996), 297-319.

[27] - On convergence rates in the central limit theorems for combinatorial structures, Europ. J. Combin. 19 (1998), 329-343. 
[28] V. Jarník, Zur metrischen Theorie der diophantischen Approximationen, Prace Mat. Fiz. 36 (1928), 91-106.

[29] —, Über die simultanen diophantischen Approximationen, Math. Z. 33 (1931), 505543.

[30] T. Kato, Perturbation Theory for Linear Operators, Springer, 1980.

[31] A. I. Khinchin, Continued Fractions, Dover, New York, 1997.

[32] A. Lasota and M. Mackey, Chaos, Fractals and Noise; Stochastic Aspects of Dynamics, Appl. Math. Sci. 97, Springer, 1994.

[33] L. Lhote, Computation of a class of continued fraction constants, in: Proc. ALENEXANALCO'04 (New Orleans), SIAM, 2004, 199-210.

[34] B. Mandelbrot, Intermittent turbulence in self-similar cascades: divergence of high moments and dimension of the carrier, J. Fluid Mech. 62 (1974), 331-358.

[35] D. Mauldin and M. Urbański, Dimensions and measures in infinite iterated function systems, Proc. London Math. Soc. (3) 73 (1996), 105-154.

[36] - - - Conformal iterated function systems with applications to the geometry of continued fractions, Trans. Amer. Math. Soc. 351 (1999), 4995-5025.

[37] Y. B. Pesin, Dimension Theory in Dynamical Systems: Contemporary Views and Applications, Chicago Lectures in Math., Univ. of Chicago Press, 1997.

[38] Y. B. Pesin and H. Weiss, A multifractal analysis of equilibrium measures for conformal expanding maps and Moran-like geometric constructions, J. Statist. Phys. 86 (1997), 233-275.

[39] J. Peyrière, Calculs de dimensions de Hausdorff, Duke Math. J. 44 (1977), 591-601.

[40] J. Shallit, Real numbers with bounded partial quotients. A survey, Enseign. Math. 38 (1992), 151-187.

[41] B. Vallée, Fractions continues à contraintes périodiques, J. Number Theory 72 (1998), 183-235.

[42] -, Dynamical analysis of a class of Euclidean algorithms, Theor. Comput. Sci. 297 (2003), 447-486.

[43] - Digits and continuants in Euclidean algorithms: Ergodic vs Tauberian theorems, J. Théor. Nombres Bordeaux 12 (2000), 531-570.

Facultad de Ingeniería

Universidad de Buenos Aires

Paseo Colón 850

Buenos Aires, Argentina

E-mail: ecesara@fi.uba.ar
CNRS UMR 6072 GREYC Université de Caen F-14032 Caen, France

E-mail: brigitte.vallee@info.unicaen.fr eda.cesaratto@info.unicaen.fr

Received on 6.4.2005

and in revised form on 21.8.2006 